
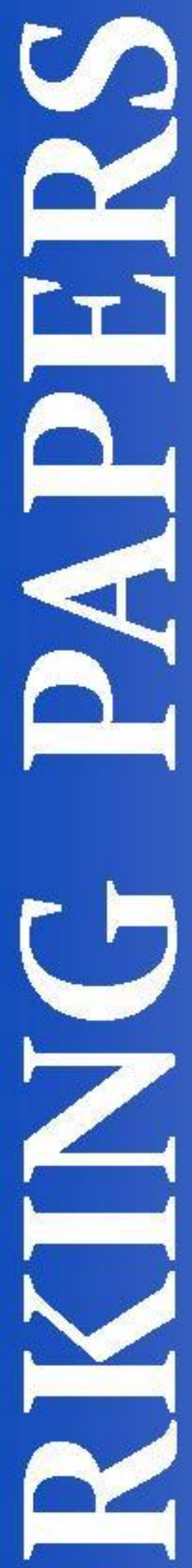

Can negative interest rates really affect option pricing? Empirical evidence from an explicitly solvable stochastic volatility model

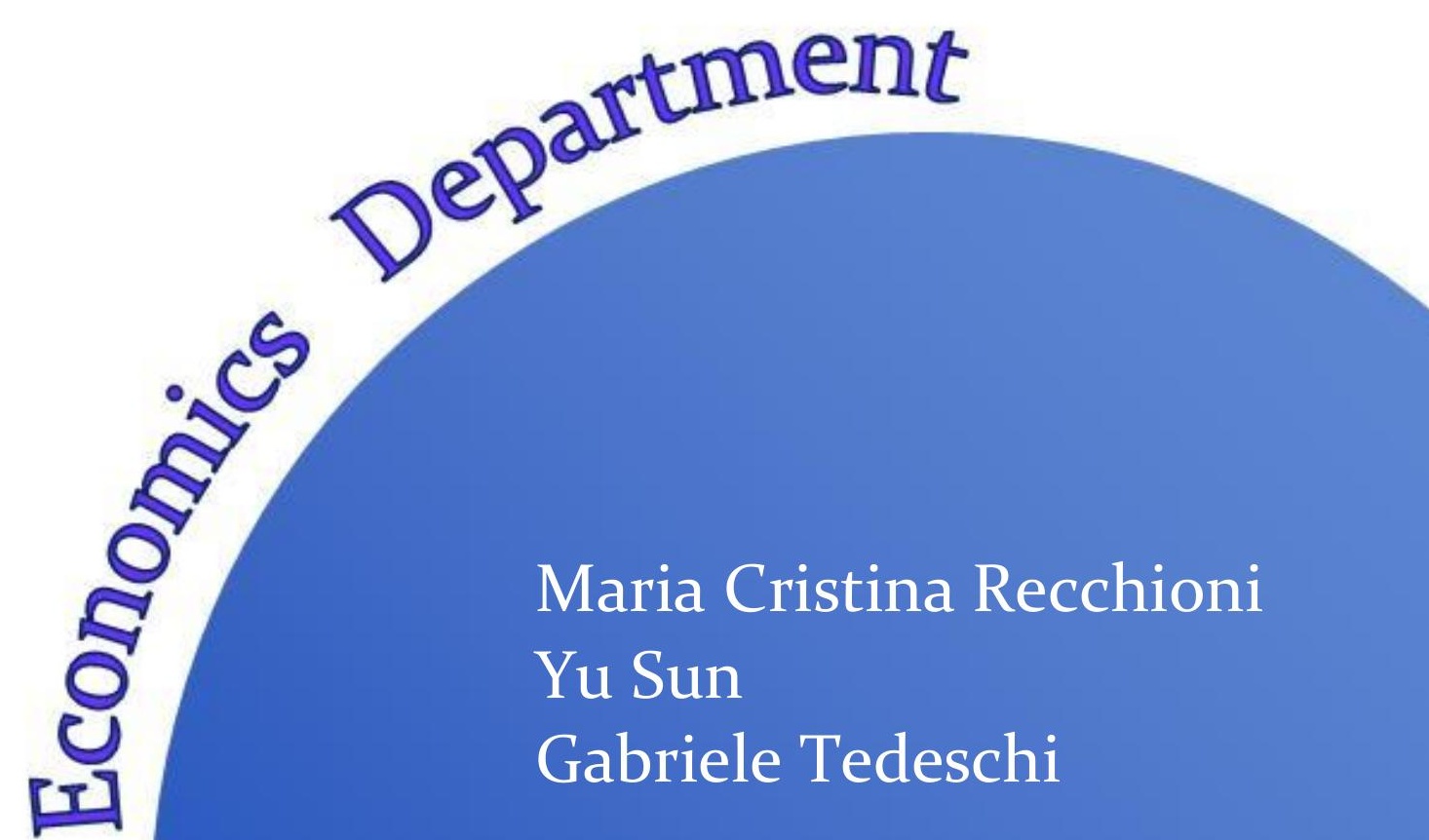

Castellón

$2016 / 23$

(Spain) 


\title{
Can negative interest rates really affect option pricing? Empirical evidence from an explicitly solvable stochastic volatility model
}

\author{
Maria Cristina Recchioni \\ Università Politecnica delle Marche \\ Department of Management \\ m.c.recchioni@univpm.it
}

\author{
Yu Sun \\ Università Politecnica delle Marche \\ Department of Management \\ y.shawn@univpm.it
}

\author{
Gabriele Tedeschi \\ Universitat Jaume I \\ Department of Economics \\ gabriele.tedeschi@gmail.com
}

\section{$2016 / 23$}

\begin{abstract}
The profound financial crisis generated by the collapse of Lehman Brothers and the European sovereign debt crisis in 2011 have caused negative values of government bond yields both in the U.S.A. and in the EURO area. This paper investigates whether the use of models which allow for negative interest rates can improve option pricing and implied volatility forecasting. This is done with special attention to foreign exchange and index options. To this end, we carried out an empirical analysis on the prices of call and put options on the U.S. S\&P 500 index and Eurodollar futures using a generalization of the Heston model in the stochastic interest rate framework. Specifically, the dynamics of the option's underlying asset is described by two factors: a stochastic variance and a stochastic interest rate. The volatility is not allowed to be negative but the interest rate is. Explicit formulas for the transition probability density function and moments are derived. These formulas are used to estimate the model parameters efficiently.

Three empirical analyses are illustrated. The first two show that the use of models which allow for negative interest rates can efficiently reproduce implied volatility and forecast option prices (i.e., S\&P index and foreign exchange options). The last studies how the U.S. three-month government bond yield affects the U.S. S\&P 500 index.
\end{abstract}

Keywords: Finance, option pricing, stochastic volatility models, calibration procedure

JEL classification: G19 


\title{
Can negative interest rates really affect option pricing? Empirical evidence from an Explicitly Solvable Stochastic Volatility Model
}

\author{
Maria Cristina Recchioni \\ Dipartimento di Management, Università Politecnica delle Marche \\ E-mail:m.c.recchioni@univpm.it \\ $\mathrm{Yu}$ Sun \\ Dipartimento di Scienze Economiche e Sociali, Università Politecnica delle Marche \\ School of Economics and Management, Nanjing University of Chinese Medicine \\ E-mail:y.shawn@univpm.it \\ Gabriele Tedeschi \\ Department of Economics, Universitat Jaume I, Castellon, Spain \\ E-mail:gabriele.tedeschi@gmail.com
}

\section{Abstract}

The profound financial crisis generated by the collapse of Lehman Brothers and the European sovereign debt crisis in 2011 have caused negative values of government bond yields both in the U.S.A. and in the EURO area. This paper investigates whether the use of models which allow for negative interest rates can improve option pricing and implied volatility forecasting. This is done with special attention to foreign exchange and index options. To this end, we carried out an empirical analysis on the prices of call and put options on the U.S. S\&P 500 index and Eurodollar futures using a generalization of the Heston model in the stochastic interest rate framework. Specifically, the dynamics of the option's underlying asset is described by two factors: a stochastic variance and a stochastic interest rate. The volatility is not allowed to be negative but the interest rate is. Explicit formulas for the transition probability density function and moments are derived. These formulas are used to estimate the model parameters efficiently.

Three empirical analyses are illustrated. The first two show that the use of models which allow for negative interest rates can efficiently reproduce implied volatility and forecast option prices (i.e., S\&P index and foreign exchange options). The last studies how the U.S. three-month government bond yield affects the U.S. S\&P 500 index. 


\section{Introduction}

This paper investigates the effect of negative short-term government bond yields on the pricing and forecasting of index options as well as foreign exchange (FX) options. Pricing and forecasting derivative products are one of the most challenging topics due to their wide use in hedging risk. The well-known stochastic models of Black Scholes 1973, Hull and White 1988, Stein and Stein 1991, and Heston 1993 assume a constant (usually positive) risk-free interest rate. However, empirical evidence has shown that time series of the U.S. and EURO short-term government bond yields have fluctuated significantly in the last fifteen years and negative values have also been seen see, for example, Jackson 2015). These facts highlight the need for two modifications. First, option pricing models with stochastic interest rate should be considered. Second, the stochastic interest rate process should allow for negative values.

We address these two points by investigating the potential of a modified version of the Heston model illustrated in Grzelak and Oosterlee 2011. Specifically, we focus on a model combining the Heston model for equity and its volatility with the Vasicek 1977 model for interest rate. Roughly speaking, the proposed model can be interpreted as the Heston multi-factor model introduced by Christoffersen, Heston, and Jacobs 2009, where one of the factors driving the option underling asset is the stochastic interest rate. The relevance of multi-factor Heston-like stochastic volatility models in efficiently describing bond yields with different maturities was highlighted in Trolle and Schwartz 2009 and Cieslak and Povala 2014. The latter propose the combined use of a short-term yield with another stochastic factor as an efficient tool to explain the yield volatility.

The model proposed here is used to investigate whether it is really necessary to use a stochastic interest rate to efficiently price and forecast options and whether these forecasts improve by allowing for negative interest rates. The benchmark models used in the empirical analysis is the Heston model with a constant risk-free interest rate and the Heston-Cox-Ingersoll-Ross (HCIR) model illustrated in Recchioni and Sun 2016. The reasons why this model outperforms the Heston model in pricing options are evident when we compare the analytical formulas for the moments of the price variable in the Heston and the modified version proposed here.

\subsection{Literature review}

Recent studies confirm the suitability of models defined by a system of stochastic differential equations (i.e., SDE models) to describe state variables such as stock, volatility and interest rate, given empirical evidence that the asset volatility and interest rate are not constant over time. The Heston model is one of the most celebrated models. In fact, thanks to the use of a stochastic

volatility, it provides accurate prices of European vanilla call and put options as well as more 
complex path-dependent options when it is assumed that the constant interest rate is realistic and that the volatility does not undergo abrupt oscillations. Indeed, local volatility models (see, for example, Dupire 1994) are also able to perfectly replicate all vanilla option prices, but this implies unrealistic spot volatility dynamics as observed by Hagan et al. 2002 and Ayache et al. 2007. Several extensions of the Heston and Dupire model, also including jumps, can be found. We mention Homescu 2014 and Itkin 2016 for an extensive overview of the literature. These extensions do not always allow for analytical treatment, but they satisfactorily replicate market data by using specific numerical approaches.

Here, we focus on models that, roughly speaking, are obtained by adding further stochastic factors to the Heston price dynamics and which allow for some analytical treatment. Examples include the models of Christoffersen et al. 2009, Fatone et al. 2009, 2013, Wong and Lo 2009, Recchioni and Sun 2016, Pacati et al. 2016, Islyaev and Date 2015, and Pun et al. 2015.

Due to the combined action of these factors, these models are able to describe the option prices and the implied volatility efficiently, even when the underlying asset is highly volatile. However, the choice of the risk-free interest rate as one of the factors driving the underlying asset is crucial when pricing options with very long maturity (i.e., maturity longer than five years such as those of some insurance contracts; see Recchioni and Sun 2016) or options in a time period where highly fluctuating short-term yields are experienced (see, for example, Jackson 2015).

Indeed, several hybrid SDE models (i.e. models obtained by correlating stochastic differential equations from different classes) have been introduced since 2000 in order to extend well-known stochastic volatility models such as the Heston model. We cite some models similar to the model proposed here. The model by Andreasen 2007 generalizes the model introduced by Zhu 2000. This generalization is obtained by using the Heston stochastic volatility model and an indirect correlation between the equity and the interest rate process. The model by Ahlip 2008 describes the spot FX rate with stochastic volatility and stochastic domestic and foreign rates. More recently, we have the so-called Schöbel-Zhu-Hull-White hybrid model illustrated by Grzelak, Oosterlee and Weeren 2012, who follow the approach proposed by Duffie, Pan and Singleton 2000 for the analytical treatment of this affine model. However, as highlighted in Grzelak and Oosterlee 2011, the model allows for negative volatility. In order to overcome this problem, they propose the use of a Cox-Ingersoll-Ross (CIR) process to describe the variance process. Finally, it is worth noting that local volatility models have also been extended to deal with stochastic interest rates (see, for example, Deelstra and Rayee 2012 and Benhamou, Gobet and Miri 2012).

The hybrid Heston model proposed here can be interpreted as a hybrid SDE model. Specifically, it is a modified version of the Heston-Hull-White (HHW) model illustrated by Grzelak and Oosterlee 2011. However, we continue to refer to this modified version as an HHW model. 


\subsection{Description of the results}

The contribution of this paper is twofold. First, we modify the multi-factor Heston model proposed in Recchioni and Sun 2016 (HCIR hereinafter) in that we use the Vasicek model (see Vasicek 1977, Mamon 2004) for the stochastic interest rate instead of the CIR model. This choice preserves the affine structure while allowing for negative values of the interest rate. As a particular case of the Hull and White model, the Vasicek model allows us to refer to it as an HHW model. This HHW model preserves the main features of the model illustrated by Grzelak and Oosterlee 2011 and is analytically tractable, an aspect that allows us to derive the probability density function of the stochastic process by solving the backward Kolmogorov equation through the same approach introduced in Recchioni and Sun 2016. The approach is based on a suitable parametrization of the probability density function, which allows us to derive elementary formulas for the moments of the asset price variable and to express the prices of European call and put options as one-dimensional integrals through an efficient approximation of the discount factor. The performance of this approximation is measured using the explicit formula (30) for the zero coupon bond in the Vasicek model.

Second, we calibrate the model in order to measure its performance in interpreting and forecasting European call and put option prices (see the empirical analysis on the U.S. S\&P 500 index options in Subsection 4.1 and on FX options on the EUR/USD exchange rate in Subsection 4.2). The calibration procedure in the empirical analysis illustrated in Subsections 4.1 and 4.2 is based on the solution to a nonlinear constrained optimization problem, whose objective function measures the relative squared difference between the observed and theoretical implied volatilities associated with call and put options. The results show that the hybrid model is able to reproduce European call and put in-sample and out-of-sample option prices with only one set of model parameters. Satisfactory out-of-sample approximations for the implied volatility for both call and put options are obtained.

Finally, we investigate the model's ability to capture the relationship between the S\&P 500 index and the U.S. three-month government bond yield and to forecast the index via the expected value conditioned on the last observation of the index itself and the bond yield. The results of this empirical analysis are preliminary and deserve further investigation.

\subsection{Outline of the paper}

The paper is organized as follows. In Section 2, we illustrate the hybrid Heston-Hull-White model and some relevant formulas. In Section 3, we propose formulas to approximate the European vanilla call and put option prices as one-dimensional integrals of explicitly known functions based 
on a simple approach to approximate the stochastic discount factor. In Section 4, we illustrate three empirical analyses. Specifically, in the first two cases we use call and put option prices as data. Consequently, we estimate the model parameters by solving constrained optimization problems whose objective functions involve the implied volatility associated with these option prices. The first empirical analysis uses the U.S. S\&P 500 index from April 2, 2012 to July 2, 2012 and the prices of the corresponding European call and put options with expiry date March 16, 2013. The second deals with the EUR/USD futures prices with maturity September 16, 2011, and the daily prices of the corresponding European call and put options with expiry date September 9, 2011. These data are observed in the time period September 27, 2010 to July 19, 2011. The performance of the model is tested by comparing the observed option prices and the out-of-sample option prices obtained using the calibrated model. The last empirical analysis investigates the dependence of the U.S. index on the U.S. three-month government bond yield. The model parameters are estimated using a pseudo-likelihood approach. The results obtained are validated by forecasting the U.S. S\&P 500 index using the explicit formula for the first-order moment of the price variable and employing the estimated parameters. Some conclusions are drawn in Section 5. The Appendix is reserved for derivations of the formula for the probability density function and the explicit formulas of the moments.

\section{Multi-factor Heston-Hull-White Model}

In this section, we present some results of the analytical treatment of the generalized version of the Heston model with a stochastic interest-free rate described by the Vasicek model as illustrated in Grzelak et.al 2011, 2012, and Guo et.al, 2013. As mentioned in the previous section, this model can also be interpreted as a multi-factor Heston model where one of the stochastic factors is the interest rate. As shown in the empirical analysis, the use of the one-factor Vasicek model makes the proposed multi-factor model more appropriate in interpreting market prices in a period where negative interest rates are seen.

In fact, the Vasicek model allows for negative values of the interest rate, which has, until now, been considered a weakness of this model. However, the negative values of the U.S. short-term government bond yields (see Figure 3 (b)) and those of German government bond yields observed in recent years give this model new appeal. We therefore consider the following generalization of the Heston model (i.e. the HHW model):

$$
\begin{aligned}
& d S_{t}=S_{t} r_{t} d t+S_{t} \sqrt{v_{t}} d W_{t}^{v}+S_{t} \Delta \sqrt{v_{t}} d Z_{t}^{v}+S_{t} \Omega d W_{t}^{r}, \quad t>0 \\
& d v_{t}=\chi\left(v^{*}-v_{t}\right) d t+\gamma \sqrt{v_{t}} d Z_{t}^{v}, \quad t>0, \\
& d r_{t}=\lambda\left(\theta-r_{t}\right) d t+\eta d Z_{t}^{r}, \quad t>0
\end{aligned}
$$


where $\Delta$ and $\Omega$ are non-negative constants and $\chi, v^{*}, \gamma, \lambda, \theta, \eta$ are positive constants, while $W_{t}^{v}$, $W_{t}^{r}, Z_{t}^{v}, Z_{t}^{r}$ are standard Wiener processes. All correlations among the Wiener processes are zero except for the following:

$$
\begin{aligned}
& E\left(d W_{t}^{v} d Z_{t}^{v}\right)=\rho_{v} d t, t>0, \\
& E\left(d W_{t}^{r} d Z_{t}^{r}\right)=\rho_{r} d t, t>0,
\end{aligned}
$$

where the quantities $\rho_{v}, \rho_{r} \in(-1,1)$ are constant correlation coefficients. Furthermore, we assume that the Feller condition, $2 \chi v^{*} / \gamma^{2}>1$, holds.

The HHW model (1)-(3) is inspired by the reformulated Heston model illustrated in Grzelak et al. 2011 (see, Eqs. (2.9)-(2.10) in Section 2.2 of that paper). The main difference is the choice of the term $S_{t} \Omega d W_{t}^{r}$. In fact, the model (1)-(3) is a multi-factor model where one factor is the stochastic interest rate and the other is the stochastic volatility. This results from the fact that $\Omega$ is constant (independent of $v_{t}$ ) and that Eq. (5) holds with a constant correlation coefficient. In contrast, Grzelak et al. 2011 choose $\Omega$ dependent on $\sqrt{v_{t}}$ in order to reduce their model to the Heston model (i.e., only the stochastic factor $v_{t}$ drives the asset price) with a stochastic interest rate (see Eq. (2.1) and Lemma 2.1 in Grzelak et al. 2011). In Grzelak et al. 2011, the choice of $\Omega$ makes the extension not analytical tractable and two suitable approximations of the covariance term are therefore proposed in order to derive an explicit formula for the corresponding characteristic function. As mentioned in the Introduction, the HHW model (1)-(3) differs from the HCIR model in Recchioni and Sun 2016 due to the different dynamics of the interest rate: Vasicek dynamics in this model and CIR in the other.

The system of equations (1)-(3) is equipped with the following initial conditions:

$$
S_{0}=S_{0}^{*}, \quad v_{0}=v_{0}^{*}, \quad r_{0}=r_{0}^{*}
$$

where $S_{0}^{*}$ and $v_{0}^{*}, r_{0}^{*}$ are random variables concentrated in a point with probability one and for simplicity, these random variables are denoted by the points where they are concentrated. As specified in Heston 1991, the quantity $\chi$ is the speed of mean reversion, $v^{*}$ is the long-term mean, and $\gamma$ is the so-called volatility of volatility (vol of vol for short).

It is worth noting that the variance $v_{t}$ remains positive for any $t>0$ with probability one given that $2 \chi v^{*} / \gamma^{2}>1$ and $v_{0}=v_{0}^{*}>0$ (see Heston, 1991). As a consequence, the equity price $S_{t}$ remains positive for any $t>0$ with probability one given that $S_{0}^{*}>0$ with probability one. The HHW model allows for negative values of the interest rate, $r_{t}$, and this is a positive feature since negative short-term bond yields have recently been experienced in the U.S. and European financial markets. 
In order to deduce analytical formulas for this model, we use Ito's lemma and Eq. (1) to derive the stochastic differential equation satisfied by the log-price process, $x_{t}=\ln \left(S_{t} / S_{0}\right), t>0$ :

$$
\begin{aligned}
d x_{t} & =\left[r_{t}-\frac{1}{2}\left(\tilde{\psi} v_{t}+\Omega^{2}\right)\right] d t+\sqrt{v_{t}} d W_{t}^{v}+\Delta \sqrt{v_{t}} d Z_{t}^{v}+\Omega d W_{t}^{r}, \\
d v_{t} & =\chi\left(v^{*}-v_{t}\right) d t+\gamma \sqrt{v_{t}} d Z_{t}^{v}, \\
d r_{t} & =\lambda\left(\theta-r_{t}\right) d t+\eta d Z_{t}^{r},
\end{aligned}
$$

where $\tilde{\psi}$ in $(7)$ is given by

$$
\tilde{\psi}:=1+\Delta^{2}+2 \rho_{v} \Delta
$$

Eq. (6) implies that the process $\left(x_{t}, v_{t}, r_{t}\right)$ satisfies the following initial conditions:

$$
x_{0}=x_{0}^{*}=0, \quad v_{0}=v_{0}^{*}, \quad r_{0}=r_{0}^{*},
$$

where $x_{0}^{*}$ is a random variable that we assume to be concentrated in a point with probability one.

We now illustrate the main formulas used in the empirical analysis; their derivation can be found in the Appendix. It is worth noting that the analytical formulas deduced for the HHW model can also provide analytical formulas for the Heston model with a suitable choice of the HHW parameters. Furthermore, a comparison of the Heston and HHW formulas allows us to identify which HHW model parameters play a crucial role in interpreting option prices.

\subsection{Transition probability density function in the HHW and Heston models}

Let us start by denoting the set of real numbers by $\mathbf{R}$, the set of positive real numbers by $\mathbf{R}^{+}$, and the $n$-dimensional Euclidean vector space by $\mathbf{R}^{n}$. We now illustrate the formula for the transition probability density function (pdf for short) associated with the stochastic differential system (7), (8), (9) (i.e., the HHW model) and the pdf of the Heston model. The pdf of the HHW model has two main advantages.

The first is that it evaluates the pdf as a one-dimensional integral of a smooth, explicitly known integrand given by the product of two functions. One function depends only on the parameters $\Theta_{v}=\left(\gamma, \chi, v^{*}, \rho_{v}, \Delta\right) \in \mathbf{R}^{5}$ of the volatility process and the other depends only on the parameters $\Theta_{r}=\left(\eta, \lambda, \theta, \rho_{r}, \Omega\right) \in \mathbf{R}^{5}$ of the interest rate process.

The second advantage is that the specific parametrization used to derive the formula (i.e., the parameter $q$ appearing in the pdf given in Eq. (12)) allows some integrals appearing in the option price formulas and in the formulas for the moments of the price variable to be computed explicitly. 
Specifically, let $p_{f}$ denote the pdf associated with the stochastic differential system (7), (8), (9). The following formula then holds (see the Appendix for the proof):

$$
\begin{gathered}
p_{f}\left(x, v, r, t, x^{\prime}, v^{\prime}, r^{\prime}, t^{\prime}\right)=\frac{e^{q\left(x-x^{\prime}\right)}}{2 \pi} \int_{-\infty}^{+\infty} e^{\imath k\left(x^{\prime}-x\right)} L_{v, q}\left(t^{\prime}-t, v, v^{\prime}, k ; \Theta_{v}\right) L_{r, q}\left(t^{\prime}-t, r, r^{\prime}, k ; \Theta_{r}\right) d k, \\
(x, v, r),\left(x^{\prime}, v^{\prime}, r^{\prime}\right) \in \mathbf{R} \times \mathbf{R}^{+} \times \mathbf{R}, t, t^{\prime} \geq 0, q \in \mathbf{R}, t^{\prime}-t>0
\end{gathered}
$$

where $\imath$ is the imaginary unit and $L_{v, q}$ and $L_{r, q}$ are explicitly known functions given in Eqs. (69) and (71). The specific form of the transition probability density function in Eq. (12) is a consequence of the correlation structure (4)-(5). In Recchioni and Sun 2016, a formula for the pdf of the Heston model with a stochastic interest rate described by the CIR model, i.e., the HCIR model, was deduced. Specifically, the function $L_{v, q}$ appearing in (12) is the same one used in the HCIR model since it depends only on the volatility process, whose dynamics is the same for the HCIR and HHW models. In contrast, the function $L_{r, q}$ in the HHW model differs from the one in the HCIR model since it only depends on the specific stochastic model used to describe the interest rate process.

Formula (12) shows how the introduction of a stochastic interest rate as a factor driving the asset price dynamics can affect the corresponding option prices. That is, we use formula (12) to compare the probability density functions of the Heston and HHW models. In fact, the pdf, $p_{f}^{H}$, of the Heston model is given by

$$
\begin{gathered}
p_{f}^{H}\left(x, v, t, x^{\prime}, v^{\prime}, t^{\prime}\right)=\frac{e^{q\left(x-x^{\prime}\right)}}{2 \pi} \int_{-\infty}^{+\infty} e^{\imath k\left(x^{\prime}-x\right)} L_{v, q}\left(t^{\prime}-t, v, v^{\prime}, k ; \Theta_{v}\right) e^{Q_{r, q}^{H}\left(t^{\prime}-t, r, k\right)} d k, \\
(x, v, r),\left(x^{\prime}, v^{\prime}, r^{\prime}\right) \in \mathbf{R} \times \mathbf{R}^{+} \times \mathbf{R}, t^{\prime}>t \geq 0, q \in \mathbf{R},
\end{gathered}
$$

where $L_{v, q}$ is the same function appearing in (12) and whose expression is given in Appendix Eq. (69), while the function $Q_{r, q}^{H}$ is given by

$$
Q_{r, q}^{H}\left(t^{\prime}-t, r, k\right)=-r(\imath k-q)\left(t^{\prime}-t\right), t^{\prime}>t, r, k \in \mathbf{R} .
$$

A comparison of formulas (12) and (13) shows that the main difference between the Heston and the HHW models lies in the two terms $L_{r, q}$ and $e^{Q_{r, q}^{H}}$, respectively, and that $L_{r, q}$ reduces to $e^{Q_{r, q}^{H}}$ in the limit $\Omega \rightarrow 0^{+}, \eta \rightarrow 0^{+}$and $\lambda \rightarrow 0^{+}$. We investigate this difference further in Section 2.2 .

We conclude this section by noting that the initial stochastic volatility is not observable in the financial market and is usually estimated by an appropriate calibration (see, for example, Bühler, 2002). The choice of the initial stochastic interest rate has no well-established procedure. In fact, the best proxy for the short-term rate under the risk-neutral measure has not been identified yet.

Given this, we estimate the initial stochastic interest rate in the empirical analyses illustrated in Sections 4.1 and 4.2 but we do not estimate the initial stochastic rate in Section 4.3 where we 
calibrate the model under the physical measure by using only the index values and the short-term government bond yields as data.

\subsection{Moments of the price variable in the $\mathrm{HHW}$ model}

As shown in Eqs. (83), (84) (see the Appendix), the explicit formulas for the expected values $\mathcal{M}_{m}$ and $\mathcal{M}_{m}^{H}$ of the integer powers of the asset price conditioned on the observations at time $t=0$ in the HHW and Heston models are, respectively:

$$
\mathcal{M}_{m}\left(t^{\prime}\right)=E\left(S_{t^{\prime}}^{m}\right)=S_{0}^{m} e^{Q_{v, m}\left(t^{\prime}, v_{0}, 0 ; \Theta_{v}\right)} e^{Q_{r, m}\left(t^{\prime}, r_{0}, 0 ; \Theta_{r}\right)}
$$

and

$$
\mathcal{M}_{m}^{H}\left(t^{\prime}\right)=E\left(S_{t^{\prime}}^{m}\right)=S_{0}^{m} e^{Q_{v, m}\left(t^{\prime}, v_{0}, 0 ; \Theta_{v}\right)} e^{m r_{0} t^{\prime}}
$$

where $Q_{v, m}$ and $Q_{r, m}$ are given by formulas (74) and (75) evaluated at $k=0$ (see the Appendix). Specifically, we have:

$$
Q_{v, m}\left(t^{\prime}, v_{0}, 0 ; \Theta_{v}\right)=-2 \frac{\chi v^{*}}{\gamma^{2}} \ln \left(s_{m, v, b} /\left(2 \zeta_{m, v}\right)\right)-2 \frac{\chi v^{*}}{\gamma^{2}}\left(\zeta_{m, v}+\mu_{m, v}\right) t^{\prime}-2 \frac{v_{0}}{\gamma^{2}}\left(\zeta_{q, v}^{2}-\mu_{m, v}^{2}\right) \frac{s_{m, v, g}}{s_{m, v, b}},
$$

where $s_{m, v, b}, \zeta_{m, v}, \mu_{m, v}, s_{m, v, g}$ are obtained by evaluating Eqs. (53)-(56) (see the Appendix) at $k=0$, while $Q_{r, m}\left(t^{\prime}, r_{0}, 0 ; \Theta_{r}\right)$ has the following elementary expression:

$$
\begin{aligned}
& Q_{r, m}\left(t^{\prime}, r_{0}, 0 ; \Theta_{r}\right)=\frac{1}{2}\left(m^{2}-m\right) \Omega^{2} t^{\prime}+m\left(t^{\prime}-\Psi_{1, \lambda}\left(t^{\prime}\right)\right)\left(\frac{\lambda \theta+m \eta \Omega \rho_{r}}{\lambda}\right)+ \\
& \frac{\eta^{2}}{2} \frac{m^{2}}{\lambda^{2}}\left(t^{\prime}+\Psi_{2, \lambda}\left(t^{\prime}\right)-2 \Psi_{1, \lambda}\left(t^{\prime}\right)\right)+r_{0} m \Psi_{1, \lambda}\left(t^{\prime}\right),
\end{aligned}
$$

where $\Psi_{j, \lambda}(\tau), j=1,2, \lambda>0, \tau>0$ is

$$
\Psi_{j, \lambda}(\tau)=\frac{1-e^{-j \lambda \tau}}{j \lambda}, \tau>0
$$

When negative values of the interest rate (i.e., $\left.r_{0}<0\right)$ are allowed, the moments of the Heston model (see Eq. (16)) approach zero as $t^{\prime}$ goes to $+\infty$. In other words, negative values of the interest rate, $r_{0}$, imply, on average, a fall in the price. However, this finding is not coherent with the empirical evidence (see Figure 2). The HHW model behaves in a very different way. In fact, negative values of $r_{0}$ do not necessarily imply decreasing price moments, especially for long time horizons. This is due to the fact that the long-term behavior of $\mathcal{M}_{m}\left(t^{\prime}\right)$ is independent of $r_{0}$ while it does depend on the following quantity:

$$
m\left(\frac{\lambda \theta+m \eta \Omega \rho_{r}}{\lambda}\right)+\frac{1}{2}\left(m^{2}-m\right) \Omega^{2}+\frac{\eta^{2}}{2} \frac{m^{2}}{\lambda^{2}}=m \theta-\frac{\Omega^{2}}{2} m+\frac{m^{2}}{2}\left(1-\rho_{r}^{2}\right) \Omega^{2}+\frac{m^{2}}{2}\left(\Omega \rho_{r}+\frac{\eta}{\lambda}\right)^{2} .
$$


The sign of this quantity depends on the correlation coefficient $\rho_{r}$. That is, nonnegative values of $\rho_{r}$ guarantee positive values of price moments for sufficiently large values of $t^{\prime}$, while negative values of $\rho_{r}$ with small values of $\theta$ may anticipate a decrease in the price.

A nice feature of this model is that negative values of $r_{0}$ play a crucial role only in short and medium time horizons. This behavior is plausible since the market expectation on long-term interest rates is expected to be positive in a "calm" financial climate.

Let us further analyze this issue by focusing on the first order moment of the price in the two models. Using formula (16) and setting $m=1$ in the Heston model, we obtain the following formula for the expected value of the asset price conditioned on the observations at $t=0$ of price, variance and interest rate:

$$
E^{H}\left(S_{t^{\prime}}\right)=S_{0} e^{r_{0} t^{\prime}}
$$

Similarly, using formula (15), we obtain the following formula for the expected value in the HHW model:

$$
E\left(S_{t^{\prime}}\right)=S_{0} e^{r_{0} \Psi_{1, \lambda}\left(t^{\prime}\right)+\theta\left(t^{\prime}-\Psi_{1, \lambda}\left(t^{\prime}\right)\right.} e^{\left(t^{\prime}-\Psi_{1, \lambda}\left(t^{\prime}\right)\right) \frac{\eta^{2}}{2 \lambda^{2}}} e^{-\frac{\eta^{2}}{4 \lambda} \Psi_{1, \lambda}^{2}\left(t^{\prime}\right)} e^{\left(t^{\prime}-\Psi_{1, \lambda}\left(t^{\prime}\right)\right) \frac{\eta}{\lambda} \Omega \rho_{r}}
$$

Some comments on formulas (20) and (21) are warranted. Following the approaches illustrated in Mamon 2004 to derive the bond price in the Vasicek model, we obtain:

$$
E\left(e^{\int_{0}^{t^{\prime}} r_{u} d u} \mid r_{t=0}=r_{0}\right)=e^{r_{0} \Psi_{1, \lambda}\left(t^{\prime}\right)+\theta\left(t^{\prime}-\Psi_{1, \lambda}\left(t^{\prime}\right)\right)} e^{-\frac{\eta^{2}}{4 \lambda} \Psi_{1, \lambda}^{2}\left(t^{\prime}\right)+\frac{\eta^{2}}{2 \lambda^{2}}\left(t^{\prime}-\Psi_{1, \lambda}\left(t^{\prime}\right)\right)}
$$

so formula (21) can be rewritten as follows:

$$
\begin{aligned}
& E\left(S_{t^{\prime}}\right)=S_{0} e^{\left(t^{\prime}-\Psi_{1, \lambda}\left(t^{\prime}\right)\right)\left(\frac{\eta}{\lambda} \Omega \rho_{r}\right)} e^{r_{0} \Psi_{1, \lambda}\left(t^{\prime}\right)+\theta\left(t^{\prime}-\Psi_{1, \lambda}\left(t^{\prime}\right)\right)} e^{\left(t^{\prime}-\Psi_{1, \lambda}\left(t^{\prime}\right)\right) \frac{\eta^{2}}{2 \lambda^{2}}} e^{-\frac{\eta^{2}}{4 \lambda} \Psi_{1, \lambda}^{2}\left(t^{\prime}\right)} \\
& =S_{0} e^{\frac{\eta}{\lambda} \Omega \rho_{r}\left(t^{\prime}-\Psi_{1, \lambda}\left(t^{\prime}\right)\right)} E\left(e^{t_{0}^{t^{\prime}} r_{u} d u} \mid r_{t=0}=r_{0}\right)
\end{aligned}
$$

Formula (23) shows how the direct correlation, $\rho_{r}$, between asset price and interest rate affects the expected value. In the empirical analysis of Section 4.3, we analyze the potential of formula (23) in forecasting the U.S. S\&P 500 index.

\section{Integral formulas to price European vanilla call and put options in the HHW model}

In this section, we deduce option pricing formulas for European vanilla call and put options with strike price, $E$, and maturity, $T$. We start with

$$
C\left(S_{0}, T, E, r_{0}, v_{0}\right)=E^{Q}\left(e^{-\int_{0}^{T} r_{t} d t}\left(S_{0} e^{x_{T}}-E\right)_{+}\right), S_{0}, T, v_{0}>0, r_{0} \in \mathbf{R}
$$


and

$$
P\left(S_{0}, T, E, r_{0}, v_{0}\right)=E^{Q}\left(e^{-\int_{0}^{T} r_{t} d t}\left(E-S_{0} e^{x_{T}}\right)_{+}\right), S_{0}, T, E, v_{0}>0, r_{0} \in \mathbf{R},
$$

where $(\cdot)_{+}=\max \{\cdot, 0\}$, and the expectation is taken under the risk-neutral measure $Q$ (see, for example, Duffie, 2001, Schoutens, 2003) in the model (1), (2), (3). In the two empirical analyses involving option prices, we calibrate the model only with option price data. In this way we avoid the introduction of risk premia because only the risk-neutral measure and not the physical measure is involved.

The option prices in formulas (24) and (25) require the evaluation of a three-dimensional integral whose accurate numerical evaluation is very time consuming. However, features in the HHW model allow the computation of these three-dimensional integrals to be reduced to the numerical evaluation of one-dimensional integrals.

A challenging task in evaluating European call and put option prices is the approximation of the stochastic integral defining the discount factor (see Eqs. (24), (25)). We approximate the integral using a quadrature rule where the weights are chosen considering the features of the stochastic interest rate model used. This approach is inspired by the work of Choi and Wirjanto 2007 and suggests the following approximation:

$$
e^{-\int_{0}^{T} r(t) d t} \approx e^{-r_{0}(1-\omega) T} e^{-r_{T} \omega T}
$$

where $\omega$ is chosen in order to obtain a satisfactory approximation of the bond price. Specifically, we choose

$$
\omega=\frac{1}{T} \frac{\left(T-\Psi_{1, \lambda}(T)\right)}{\lambda \Psi_{1, \lambda}(T)}
$$

where $\Psi_{1, \lambda}$ is given in (19). This guarantees that $\omega$ is positive and less than or equal to one (i.e., $0 \leq \omega \leq 1$ ) for any $T>0$. Using formulas (26) and (27), we obtain the following approximation of the bond price:

$$
E^{Q}\left(e^{-\int_{0}^{T} r_{u} d u} \mid r_{t=0}=r_{0}\right) \approx B_{A}\left(r_{0}, T\right)
$$

with

$$
B_{A}\left(r_{0}, T\right)=e^{-r_{0} \Psi_{1, \lambda}(T)-\theta\left(T-\Psi_{1, \lambda}(T)\right)} e^{\frac{\eta^{2}}{2 \lambda^{2}} \Psi_{2, \lambda}(T)} e^{\frac{\eta^{2}}{2 \lambda^{2}}\left(T-2 \Psi_{1, \lambda}(T)\left(\frac{T \Psi_{2, \lambda}(T)}{\Psi_{1, \lambda}^{2}(T)}\right)\right)}
$$

which favorably compares with the expression of the bond price, $B\left(r_{0}, T\right)$, in the Vasicek model (see, for example, Mamon 2004):

$$
B\left(r_{0}, T\right)=E\left(e^{-\int_{0}^{T} r_{u} d u} \mid r_{t=0}=r_{0}\right)=e^{-r_{0} \Psi_{1, \lambda}(T)-\theta\left(T-\Psi_{1, \lambda}(T)\right)} e^{\frac{\eta^{2}}{2 \lambda^{2}} \Psi_{2, \lambda}(T)} e^{\frac{\eta^{2}}{2 \lambda^{2}}\left(T-2 \Psi_{1, \lambda}(T)\right)} .
$$


It is worth noting that formula (29) reduces (30) when the term $\frac{T \Psi_{2, \lambda}(T)}{\Psi_{1, \lambda}^{2}(T)}$ is equal to one. This happens when $\lambda T \rightarrow 0$ since $\frac{T \Psi_{2, \lambda}(T)}{\Psi_{1, \lambda}^{2}(T)}=\frac{T \lambda}{2} \frac{1}{\tanh (T \lambda / 2)}$. In addition, the relative error of the bond approximation is given by the following simple formula:

$$
E_{\text {Bond }}=\frac{\left|B\left(r_{0}, T\right)-B_{A}\left(r_{0}, T\right)\right|}{B\left(r_{0}, T\right)}=\left|1-e^{-\frac{\eta^{2}}{\lambda^{2}} \Psi_{1, \lambda}(T)\left(\frac{T \lambda / 2}{\tanh (T \lambda / 2)}-1\right)}\right| .
$$

Figure 1 shows the true and approximated bond price (left panel) and the relative error (right panel) as a function of the maturity, $T$, when $\eta=0.005, \lambda=2.88, \theta=0.4, r_{0}=-0.7$ (dotted line) and $\eta=0.01, \lambda=3.8, \theta=0.02, r_{0}=-0.1$ (solid line). The choice of these model parameters is related to the results of the empirical analyses illustrated in Sections 4.1 and 4.2. The relative errors, shown in the right panel of Figure 1, guarantee that the bond approximation has at least four correct significant digits.
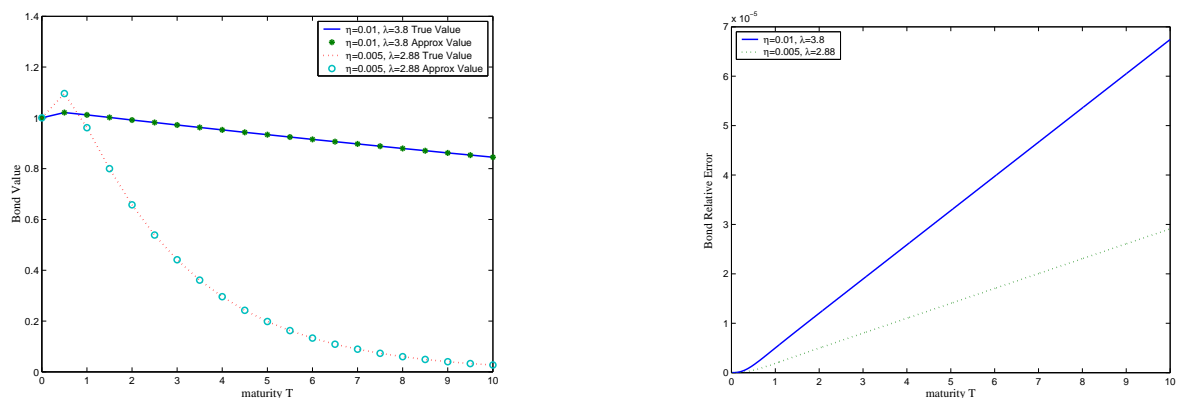

Figure 1: True and approximated bond price (left panel) and relative error of bond approximation (right panel) as a function of maturity when $\eta=0.005, \lambda=2.88, \theta=0.4, r_{0}=-0.7$ (dotted line) and $\eta=0.01, \lambda=3.8$, $\theta=0.02, r_{0}=-0.1$ (solid line).

From Equations (24), (25), (26), and (76) in the Appendix, we obtain the following approximation, $V_{A}$, for European vanilla call and put options:

$$
\begin{gathered}
V_{A}\left(S_{0}, T, E, r_{0}, v_{0}\right)=e^{-\left(\theta T+\left(r_{0}-\theta\right) \Psi_{1, \lambda}(T)\right)} e^{(\omega T)^{2} \frac{\eta^{2}}{2} \Psi_{2, \lambda}(T)} \times \\
\frac{S_{0}}{2 \pi} \int_{-\infty}^{+\infty} \frac{\left(\frac{S_{0}}{E}\right)^{(q-1-\imath k)} e^{Q_{v, q}\left(T, v_{0}, k ; \Theta_{v}\right)} e^{Q_{r, q}\left(T, r_{0}, k ; \Theta_{r}\right)}}{-k^{2}-(2 q-1) \imath k+q(q-1)} e^{(\imath k-q) \omega T\left(\Omega \rho_{r} \eta \Psi_{1, \lambda}(T)+\frac{\eta^{2}}{\lambda}\left(\Psi_{1, \lambda}(T)-\Psi_{2, \lambda}(T)\right)\right)} d k \\
T>0, S_{0}, v_{0}>0, q>1(\text { call }), \mathrm{q}<-1 \text { (put)}
\end{gathered}
$$

where functions $Q_{v, q}$ and $Q_{r, q}$ are those given in (74), (75) (see the Appendix). Formula (31) implies that the call option price differs from the put option price only in the choice of $q$, that is, $V_{A}=C_{A}$ for $q>1$ while $V_{A}=P_{A}$ for $q<-1$. This is a consequence of the parametrization used for the pdf (see Eq. (12)), which allows the integral in the $x^{\prime}$ variable to be computed explicitly for suitable values of $q$. 
Let us illustrate this for the call options. Using (26) and (76) in (24) we obtain:

$$
\begin{gathered}
C_{A}\left(S_{0}, T, E, r_{0}, v_{0}\right)=e^{-r_{0}(T-\omega T)} \int_{-\infty}^{+\infty}\left(S_{0} e^{x^{\prime}}-E\right)_{+} \int_{-\infty}^{+\infty} e^{-r^{\prime} \omega T} D_{v, q}\left(0, v_{0}, r_{0}, 0, x^{\prime}, r^{\prime}, T\right) d r^{\prime} d x^{\prime} \\
=e^{-r_{0}(T-\omega T)} \int_{-\infty}^{+\infty} e^{Q v, q\left(T, v, k ; \Theta_{v}\right)} G_{q}\left(k, S_{0}, E\right)\left[\int_{-\infty}^{+\infty} e^{-\omega T r^{\prime}} L_{r, q}\left(\tau, r, r^{\prime}, k ; \Theta_{r}\right) d r^{\prime}\right] d k,
\end{gathered}
$$

where $G_{q}$ is given by:

$$
G_{q}\left(k, S_{0}, E\right)=\int_{\ln \left(E / S_{0}\right)}^{+\infty} d x^{\prime}\left(S_{0} e^{x^{\prime}}-E\right) e^{(-q+\imath k) x^{\prime}} d x^{\prime}=\frac{S_{0}\left(\frac{S_{0}}{E}\right)^{q-1-\imath k}}{-k^{2}-(2 q-1) \imath k+q(q-1)} .
$$

The integral in Eq. (33) is convergent when $1-q<0$ and is independent of $r^{\prime}$. Thus, the integrals over $x^{\prime}$ and $r^{\prime}$ appearing in formula (32) are independent of each other and Eqs. (33) and (73) are their explicit formulas. Formula (32) shows that for the put option price, the integral

appearing in (33) must be replaced with the integral $\left[\int_{-\infty}^{\ln \left(E / S_{0}\right)} d x^{\prime}\left(E-S_{0} e^{x^{\prime}}\right) e^{(-q+\imath k) x^{\prime}} d x^{\prime}\right]$. This is convergent when $q<-1$ and has the same explicit formula as in (33).

Taking the limit $\Omega \rightarrow 0^{+}, \lambda \rightarrow 0^{+}, \eta \rightarrow 0^{+}$in Eq (31), we derive the following exact formula for the price of the European call and put options in the Heston model (see also Recchioni and Sun 2016):

$$
\begin{gathered}
V_{H}\left(S_{0}, T, E, r_{0}, v_{0}\right)=e^{-r_{0} T} \frac{S_{0}}{2 \pi} \int_{-\infty}^{+\infty} \frac{\left(\frac{S_{0}}{E}\right)^{(q-1-\imath k)} e^{Q_{v, q}\left(T, v_{0}, k ; \Theta_{v}\right)} e^{-(\imath k-q) r_{0} T}}{-k^{2}-(2 q-1) \imath k+q(q-1)} d k \\
S_{0}, T, E, r_{0}, v_{0}>0, q>1(\text { call }), q<-1 \text { (put). }
\end{gathered}
$$

In Section 4, we use formula (34) with $q>1(q<-1)$ for vanilla European call (put) options in order to compare the performance of the Heston model and the HHW model proposed here in interpreting real data. It is worth noting that the integrands appearing in formulas (31) and (34) are smooth functions whose numerical integration can be carried out efficiently using a simple quadrature rule. The smoothness of the integrands is due to the specific approach used to derive them.

\section{Empirical Analysis}

In this section, we propose three empirical analyses. The first two only involve option prices so we can work under the risk-neutral measure associated with the HHW model. In the third analysis, we investigate the dependence of the asset on the short-term rate and so here we work under the physical measure. Specifically, the first two empirical analyses involve the U.S. S\&P 500 index 
as well as the Eurodollar futures prices and the corresponding European option prices. The U.S. three-month government bond index has been used as a proxy for the initial stochastic interest rate in both analyses.

We estimate the model parameters by solving an appropriate nonlinear constrained least squares problem. In detail, let $\mathbf{R}^{12}$ denote the 12-dimensional Euclidean real space. We define the set of the constraints, $\mathcal{V}$, as follows:

$$
\begin{gathered}
\mathcal{V}=\left\{\Theta=\left(\Delta, \gamma, v^{*}, \chi, \rho_{p, v}, v_{0}, \eta, \lambda, \theta, \rho_{r}, r_{0}, \Omega\right) \in \mathbf{R}^{12} \mid\right. \\
\left.\Delta, \gamma, v^{*}, \chi, v_{0}, \eta, \lambda, \theta>0, \frac{2 \chi v^{*}}{\gamma^{2}}>1, \quad-1<\rho_{v}, \rho_{r}<1\right\} .
\end{gathered}
$$

It is worth noting that the initial values $v_{0}, r_{0}$, of the variance and interest rate process are parameters to be estimated through the calibration procedure. This is motivated by the fact that $v_{0}$ and $r_{0}$ are latent variables not really observable in the market. In fact, both $v_{0}$ and $r_{0}$ refer to a risk-neutral measure and, subsequently, their values have not yet been clearly identified by the financial market. Estimation of these parameters is not new; it can be found, for example, in Bühler 2002, Fatone et al. 2009, 2013, Grzelak and Oosterlee 2011, and Recchioni and Sun 2016.

We now formulate the calibration problem necessary to carry out the empirical analysis illustrated in Sections 4.1 and 4.2. To this end we introduce some notation. Where $n_{T}$ and $n_{D}$ are two nonnegative integers, $C_{\Theta}^{A}\left(S_{t_{j}}, T_{i}, E_{i}\right), P_{\Theta}^{A}\left(S_{t_{j}}, T_{i}, E_{i}\right)$ and $C^{o}\left(S_{t_{j}}, T_{i}, E_{i}\right), P^{o}\left(S_{t_{j}}, T_{i}, E_{i}\right)$, $i=1,2, \ldots, n_{D}, j=1,2, \ldots, n_{T}$, represent the prices of the European call and put options with maturity $T_{i}$ and strike price $E_{i}$ in the HHW model and the observed option prices at time $t=t_{j}$. In the empirical analyses, we choose $T_{1}=T_{2}=\ldots=T_{n_{D}}$, while $E_{1}<E_{2}<\ldots<E_{n_{D}}$. We denote the observed and theoretical implied volatilities associated with the call and put option prices with $\Sigma^{o, C}\left(S_{t_{j}}, r, T_{i}, E_{i}\right), \Sigma^{o, P}\left(S_{t_{j}}, r, T_{i}, E_{i}\right)$ and $\Sigma_{\Theta}^{C}\left(S_{t_{j}}, T_{i}, E_{i}\right), \Sigma_{\Theta}^{P}\left(S_{t_{j}}, T_{i}, E_{i}\right), j=1,2, \ldots, n_{T}$, $i=1,2, \ldots, n_{D}$, respectively. We recall that the implied volatility $\Sigma^{o, C}\left(S, r_{F}, T, E\right)$ is defined as the solution to the following equation:

$$
C_{B S}\left(T-t, E, S_{t}, \Sigma^{o, C}\right)=C^{o}\left(T, E, S_{t}\right)
$$

where $C^{o}$ is the observed call option price and $C_{B S}$ is the Black Scholes price at time $t$ of the European call option with strike price, $E$, and maturity, $T, T>t$ (see Black and Scholes 1973, Eq. (13)) while $r_{F}$ denotes the risk-free interest rate. We choose $r_{F}$ to be the U.S. three-month government bond yield. We estimate the model parameters using the implied volatilities. This approach works satisfactorily when very deep out-of-the-money option are excluded.

Following this approach, we estimate the model parameters by solving the following nonlinear constrained optimization problem

$$
\min _{\Theta \in \mathcal{V}} F(\Theta)
$$



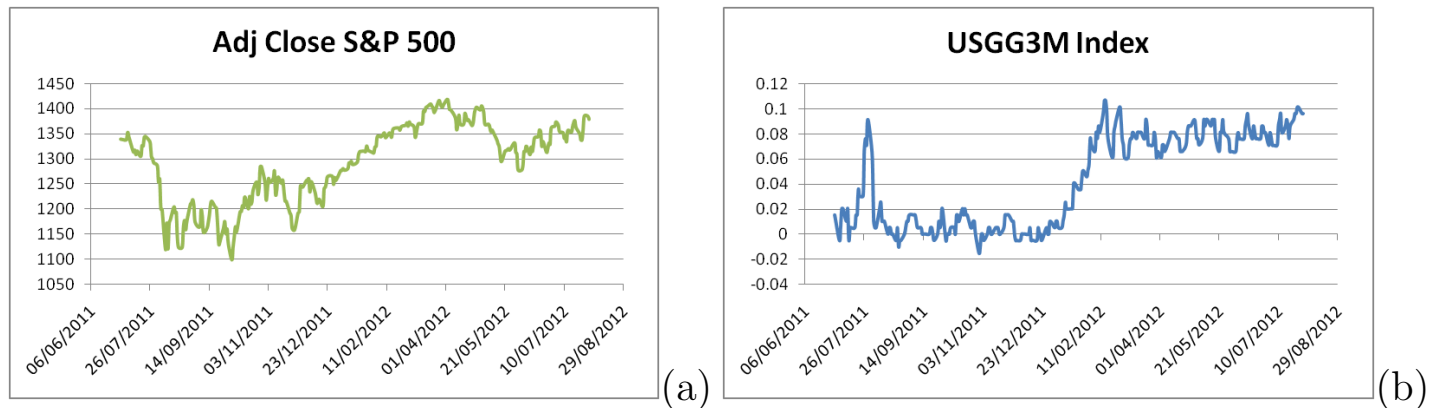

Figure 2: U.S. S\&P 500 index (a) and U.S. three-month government bond yield (in percent) (b) versus time (July 1, 2011-July 31, 2012).

where the objective function, $F_{n_{T}}$, is

$$
\begin{aligned}
& F(\Theta)=\frac{1}{n_{D} n_{T}} \sum_{j=1}^{n_{T}} \sum_{i=1}^{n_{D}}\left[\frac{\Sigma^{o, C}\left(S_{t_{j}}, r_{F}, T_{i}, E_{i}\right)-\Sigma_{\Theta}^{C}\left(S_{t_{j}}, T_{i}, E_{i}\right)}{\Sigma^{o, C}\left(S_{t_{j}}, r_{F}, T_{i}, E_{i}\right)}\right]^{2} \\
& +\frac{1}{n_{D} n_{T}} \sum_{j=1}^{n_{T}} \sum_{i=1}^{n_{D}}\left[\frac{\Sigma^{o, P}\left(S_{t_{j}}, r, T_{i}, E_{i}\right)-\Sigma_{\Theta}^{P}\left(S_{t_{j}}, T_{i}, E_{i}\right)}{\Sigma^{o, P}\left(S_{t_{j}}, r_{F}, T_{i}, E_{i}\right)}\right]^{2} .
\end{aligned}
$$

In order to show empirical evidence that the use of stochastic interest rates is crucial, we compare the proposed model with the Heston model (Heston 1993). The latter is calibrated by solving problem (37) in a feasible set that does not contain the parameters of the stochastic interest rate model except for the parameter $r_{0}$, which allows for negative values.

We use formulas (31) and (34) to evaluate option prices in the HHW and Heston model (see Recchioni and Sun 2016 for further details on the Heston pricing formulas). The one-dimensional integrals appearing in these formulas are computed using the composite midpoint quadrature rule with $2^{14}$ nodes. This quadrature rule gives satisfactory approximations since the integrands appearing in Eqs. (31) and (34) are smooth functions, which means the numerical integration does not require special care. Moreover, problem (37) is solved using a steepest descent algorithm with a variable metric (see, for example, Recchioni and Scoccia 2000, Recchioni and Sun 2016).

\subsection{U.S. S\&P 500 index options}

The empirical analysis presented in this subsection concerns the daily closing values of the U.S. S\&P 500 index and the daily closing prices of the European call and put options on this index. The expiry date of these options is March 16, 2013 and their strike prices are $E_{i}=1075+25(i-1)$, $i=1,2, \ldots, 4, E_{5}=1170$.

Figure 2(a) shows the U.S. S\&P 500 index (July 1, 2011-July 27, 2012) while Figures 3(a) and 3(b) show the corresponding call and put option prices as a function of time (April 2, 2012--July 

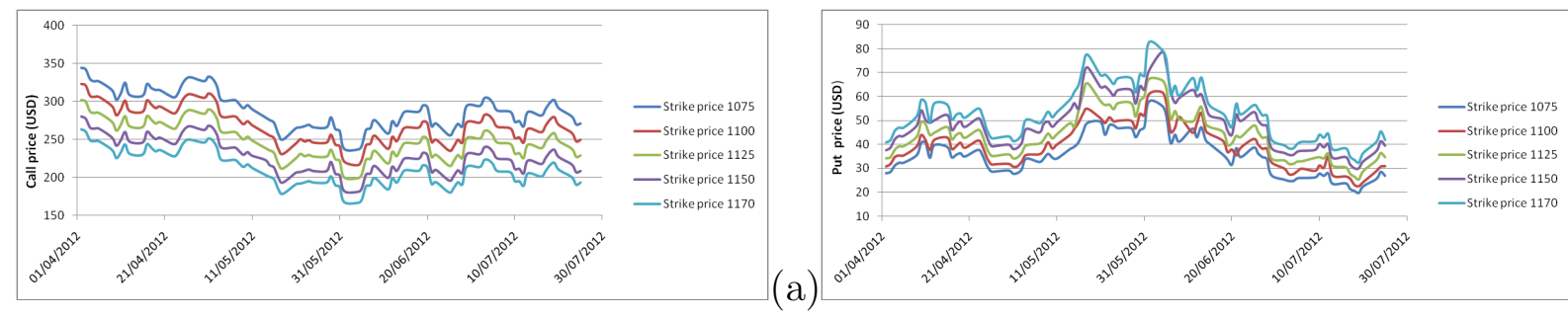

Figure 3: Prices of call (a) and put (b) options on the U.S. S\&P 500 index with strike prices $E_{i}=1075+25(i-1)$, $i=1,2, \ldots, 4$ and $E_{5}=1170$, and with expiry date $T=$ March 16, 2013, versus time.
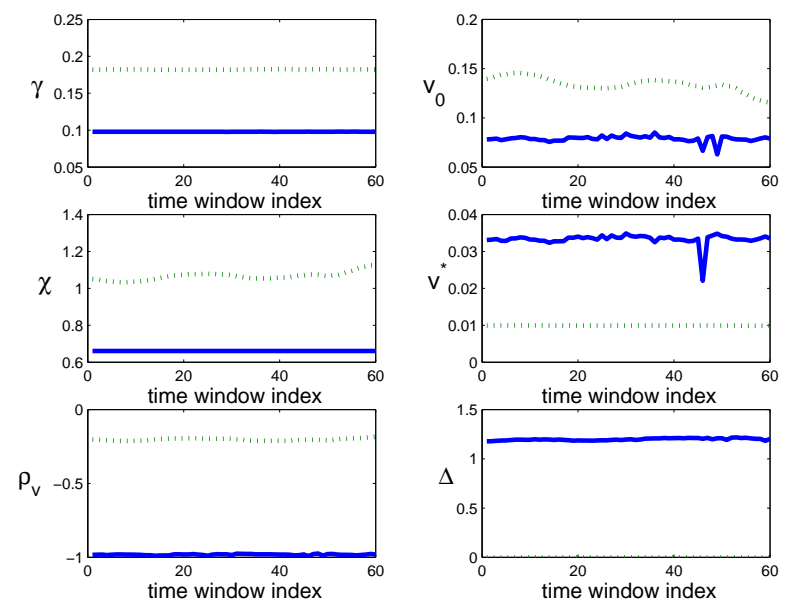

Figure 4: Estimated parameters $\gamma, v_{0}, \chi, v^{*}, \rho_{v}$, and $\Delta$ versus window index (six-day window) resulting from calibration of the HHW model (solid line) and the Heston model (dotted line).
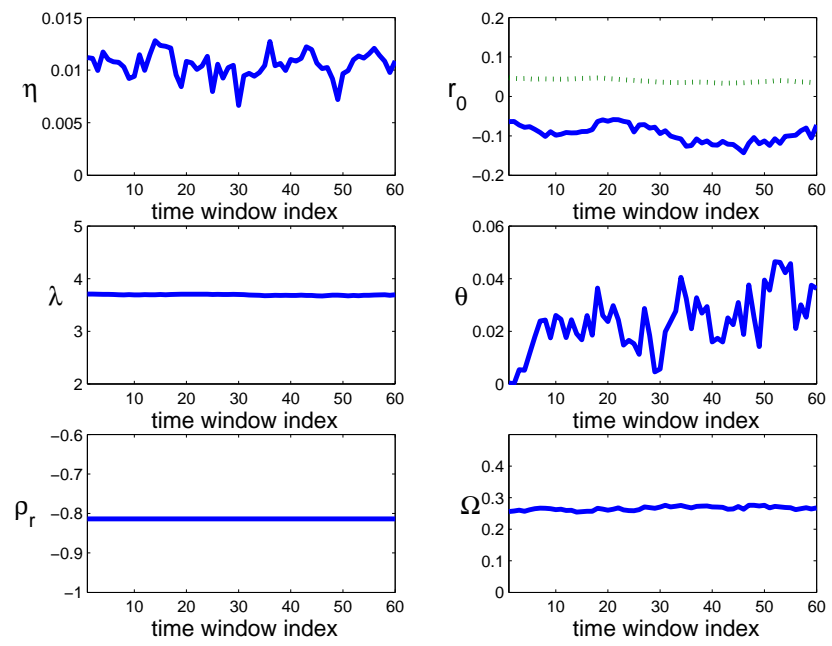

Figure 5: Estimated parameters $\eta, r_{0}, \lambda, \theta, \rho_{r}$, and $\Omega$ versus window index (six-day window) resulting from calibration of the HHW model (solid line). Note that the dotted line in the upper right panel shows the estimated values of the parameter $r_{0}$ resulting from calibration of the Heston model. 
27, 2012). Figure 2(b) shows the U.S. three-month government yields (in percent) as a function of time. These short-term bond yields are used as values of the risk-free interest rate in the Black Scholes formula in (36).
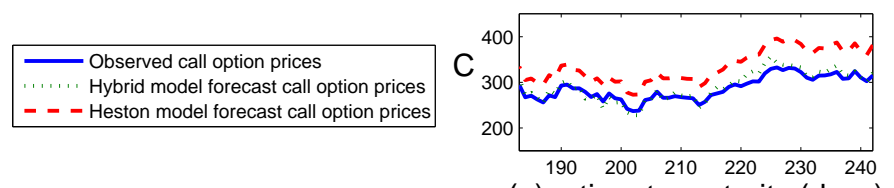

(a) time to maturity (days)
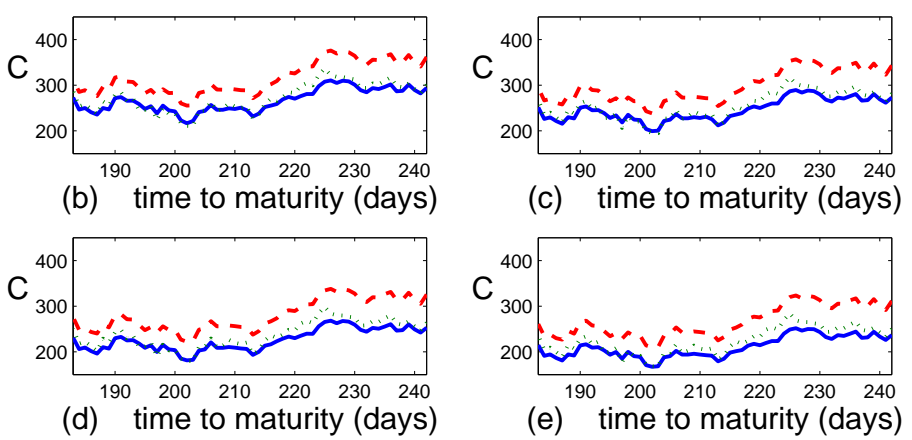

Figure 6: Observed (solid line) and in-sample call option prices (in USD) versus time to maturity (days) obtained using the HHW (dotted line) and the Heston (dashed line) models for five different strike prices: (a) $E_{1}=1075$, (b) $E_{2}=1100,(\mathrm{c}) E_{3}=1125$, (d) $E_{4}=1150$, and (e) $E_{5}=1170$.
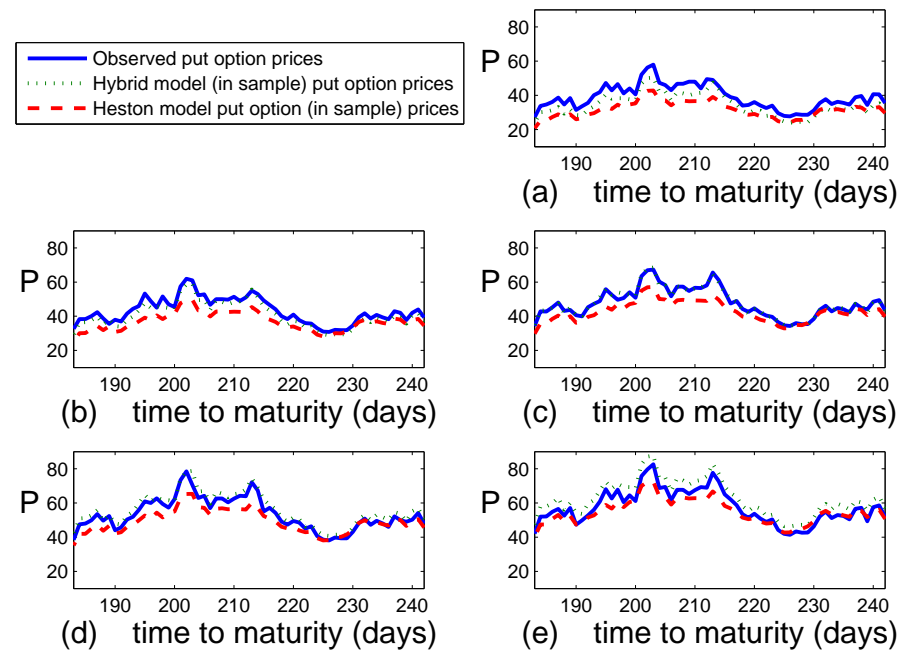

Figure 7: Observed (solid line) and in-sample put option price (in USD) versus time to maturity (days) obtained using the HHW (dotted line) and the Heston (dashed line) models for five different strike prices: (a) $E_{1}=1075$, (b) $E_{2}=1100,(\mathrm{c}) E_{3}=1125$, (d) $E_{4}=1150$, and (e) $E_{5}=1170$.

We analyze the option data using a rolling window of six consecutive trading days (i.e., $\left.n_{T}=6\right)$. As a consequence, in each window, sixty option values are used to calibrate the twelve 
parameters of the model (i.e., $n_{P}=5$ put option prices and $n_{C}=5$ call option prices for $n_{T}=6$ days). This window is moved by one day along the historical series covering the period April 2 to July 2, 2012. The calibration problems (37) solved by moving the window are $66-n_{T}$. In this way, we obtain a historical series of daily observations for each parameter. The values of the parameters obtained in the $j$-th window are representative of the last day of the $j$-th window. We highlight the fact that when the values of the estimated parameters are constant in time, the model reproduces the asset price dynamics in the period analyzed by using only one set of model parameters.

Figures 4 and 5 show the parameter values as a function of the index $j, j=1,2, \ldots, 66-n_{T}$. We observe that these values are relatively constant as a function of time.
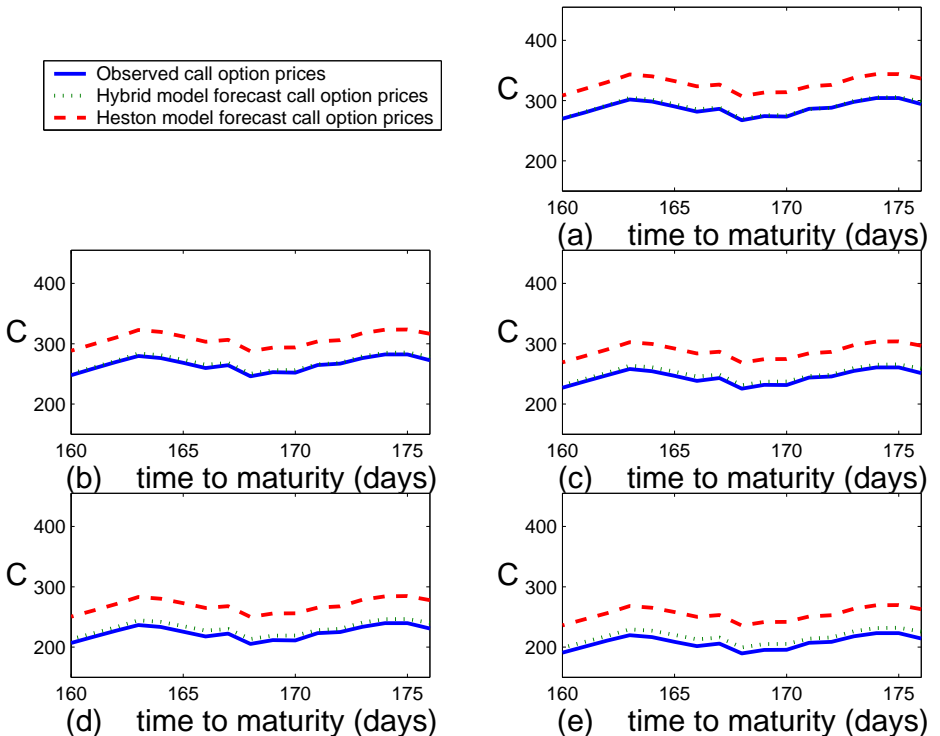

Figure 8: Observed (solid line) and out-of-sample call option price forecast (in USD) versus time to maturity (days) obtained using the HHW (dotted line) and the Heston (dashed line) models for five different strike prices: (a) $E_{1}=1075$, (b) $E_{2}=1100$, (c) $E_{3}=1125$, (d) $E_{4}=1150$, and (e) $E_{5}=1170$.

Figures 6 and 7 show the in-sample values of the European call and put options obtained using the Heston (dashed line) and the HHW (dotted line) models with the parameters estimated in the period April 2, 2012 to July 2, 2012. These figures show that the theoretical option prices of the HHW model provide satisfactory approximations of observed put prices for all strike-price values and time to maturity. These values outperform those obtained with the Heston model. In fact, the sample mean of the relative errors of the call and put options respectively are $4.1 \%$ and $6.7 \%$ for the HHW model compared to $21.2 \%$ and $11.2 \%$ for the Heston model. The HHW relative errors outperform those for the hybrid Heston-CIR model (see Recchioni and Sun 2016), which are $9.6 \%$ and $6.9 \%$ for call and put options, respectively. This is probably a consequence of the fact that in the Heston-CIR model the interest rate cannot assume negative values. This 

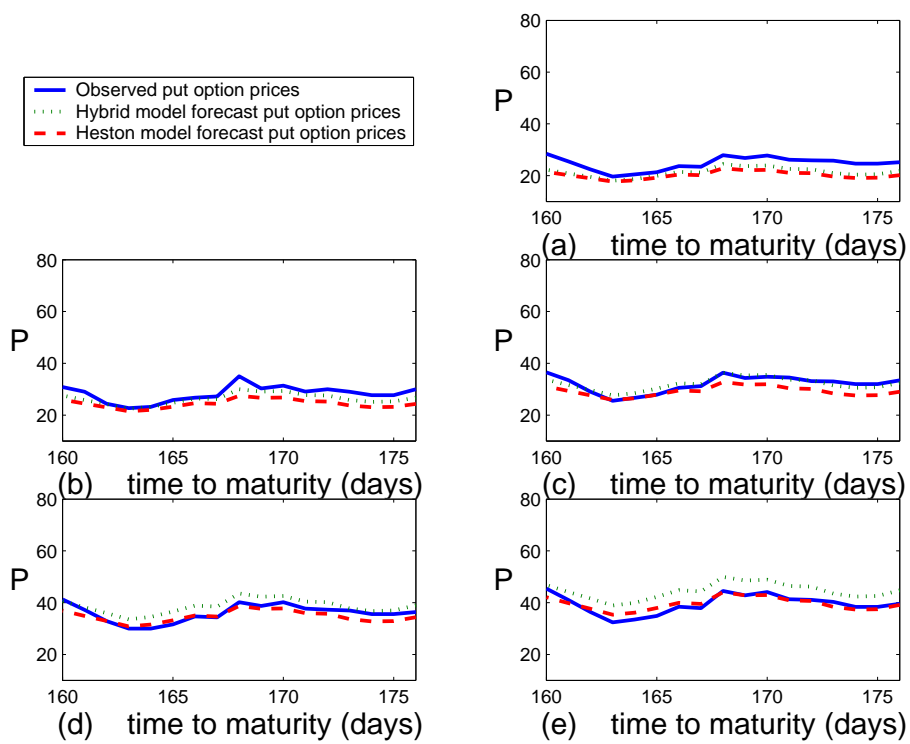

Figure 9: Observed (solid line) and out-of-sample put option price forecast (in USD) versus time to maturity (days) obtained using the HHW (dotted line) and the Heston (dashed line) models for five different strike prices: (a) $E_{1}=1075$, (b) $E_{2}=1100$, (c) $E_{3}=1125$, (d) $E_{4}=1150$, and (e) $E_{5}=1170$.

finding is confirmed by the fact that the estimated values of $r_{0}$ is on average equal to 0.0398 in the Heston model and 0.00022 in the Heston-CIR model, while it is -0.0946 in the HHW model.

Figures 6 and 7 show that the HHW model is capable of matching both call and put option prices with sufficient accuracy for several strike prices and expiry dates using only one set of parameters. This good performance is achieved through the use of a stochastic interest rate that allows for a negative rate.

We use the value of the model parameters estimated in the last window, June 25, 2012-July 2, 2012, to evaluate the out-of-sample European call and put option prices. The out-of-sample period is July 3 to July 27, 2012. The time to maturity for this period ranges from 176 to 160 days. We measure the performance of the proposed stochastic model and its parameter estimation procedure with an a posteriori validation. That is, we compare the observed out-of-sample option prices with those obtained using formulas (34), (31), which use estimated parameters and observed spot prices.

Figures 8 and 9 show the out-of-sample option prices for the HHW model (dotted line) and the Heston model (dashed line). The out-of-sample put option prices in the Heston model are very accurate while the call option prices are not. The HHW model provides accurate approximations of put option prices and outperforms the Heston model in approximating the call options. In fact, the sample mean of the relative errors on the call and put options obtained using the HHW model are $2.3 \%$ and $9.1 \%$ respectively, compared to $17.9 \%$ and $9.6 \%$ for the Heston model. The HHW relative errors outperform those for the hybrid HCIR model illustrated in Recchioni and 
Yu 2016, which are 7.8\% and 9.5\% for call and put options respectively.

In conclusion, the empirical analysis shows that the hybrid model interprets the real data considered in the period April 2 to July 27, 2012 satisfactorily using only one set of model parameters.

\subsection{FX options on EUR/USD}

In the second experiment we consider the daily values of the futures price on the EUR/USD currency exchange rate with maturity on September 16, 2011, (the third Friday of September 2011) and the daily prices of the corresponding European call and put options with expiry date September 9, 2011 and strike prices $E_{i}=1.375+0.005(i-1), i=1,2, \ldots, 18$. The strike prices $E_{i}, i=1,2, \ldots, 18$, are expressed in USD. These prices are observed in the period from September 27, 2010 to July 19, 2011. The observations are made daily and the prices considered are the closing prices of the day. Recall that a year has about 250-260 trading days and a month has about 21 trading days. Figure 10 shows the futures price EUR/USD (ticker YTU1 Currency)

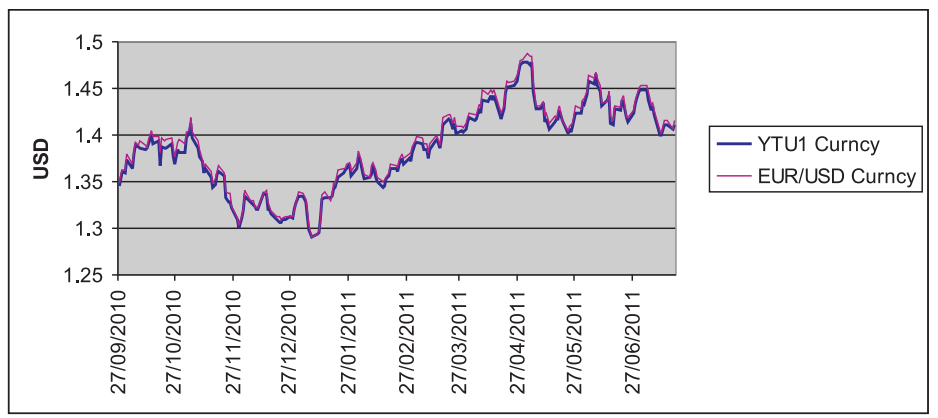

Figure 10: YTU1 (blue line) and EUR/USD currency exchange rate (pink line) versus time.

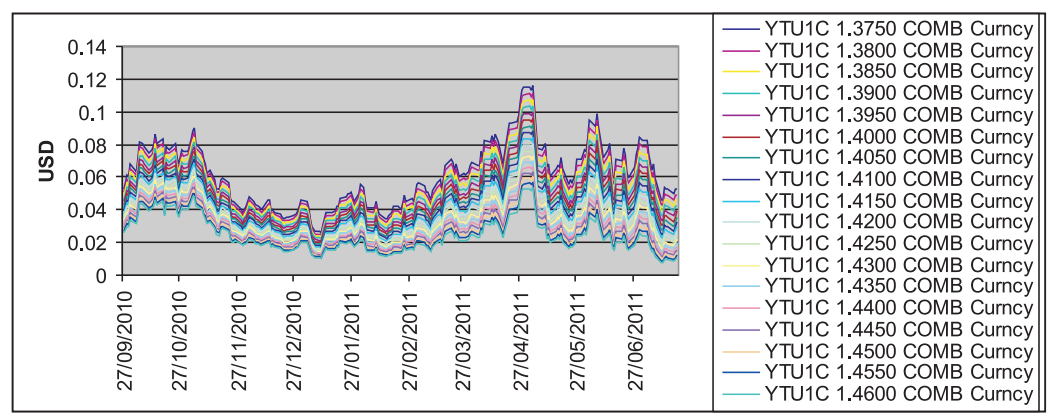

Figure 11: Call option prices on YTU1 with strike price $E_{i}=1.375+0.005(i-1), i=1,2, \ldots, 18$, and expiry date $T=$ September 9, 2011 versus time.

(blue line) and the EUR/USD currency exchange rate (pink line) as a function of time. Figures 


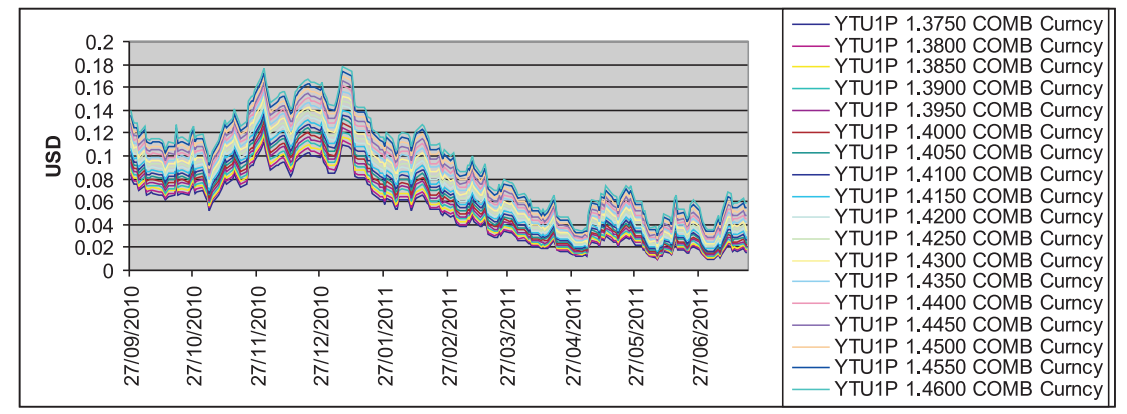

Figure 12: Put option prices on YTU1 with strike price $E_{i}=1.375+0.005(i-1), i=1,2, \ldots, 18$, and expiry date $T=$ September 9, 2011 versus time.

11 and 12 show, respectively, the prices (in USD) of the corresponding call and put options with maturity time September 9, 2011 and strike price $E_{i}, i=1,2, \ldots, 18$, as a function of time.
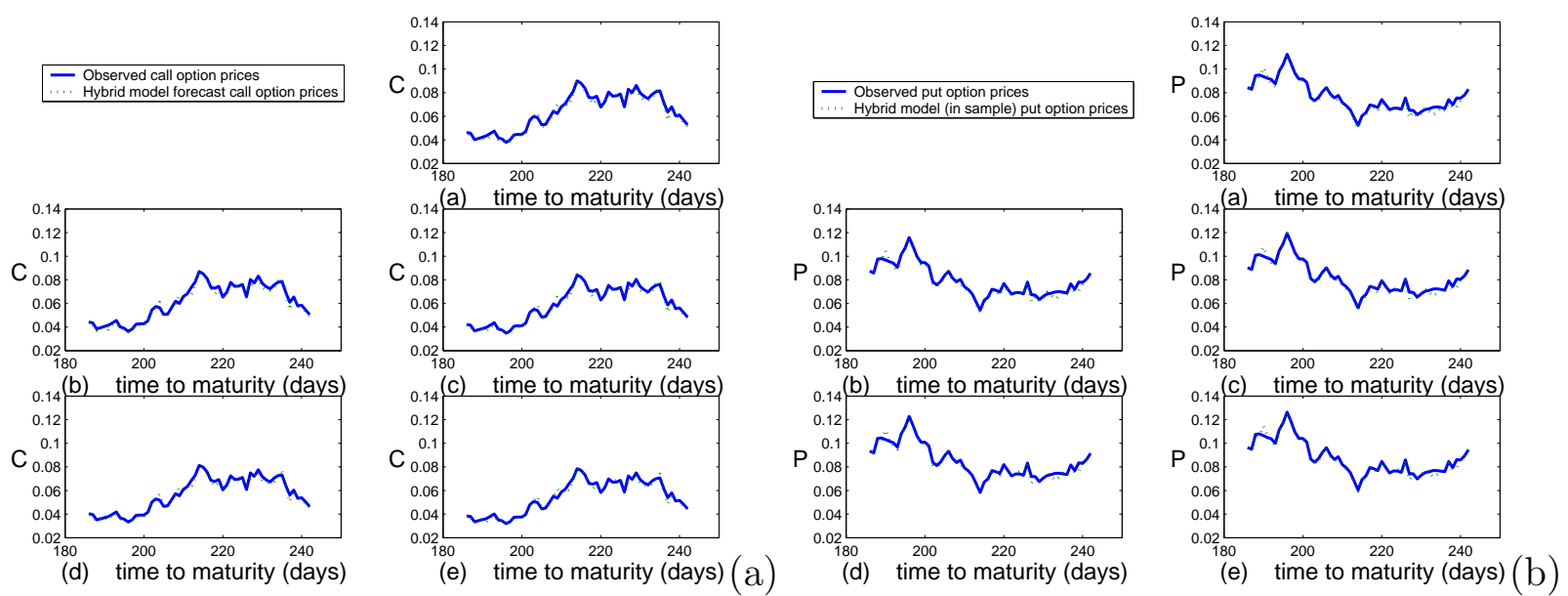

Figure 13: In-sample observed and theoretical call (a) and put (b) option prices on YTU1 with strike price $E_{i}=1.375+0.005(i-1), i=1,2, \ldots, 18$, and expiry date $T=$ September 9,2011 versus time to maturity (September 27, 2010-December 17, 2010).

We define the moneyness of an option on a given day as the ratio between the strike price of the option and the futures price on the EUR/USD exchange rate of that day.

As in the previous subsection, we consider a rolling window containing the prices of one trading day (i.e., $n_{T}=1$ ). This window is moved by one day along the historical series. The time window covers the period September 27 to December 17, 2010 and $61-n_{T}$ calibration problems are solved (see Eq. (37)). As a consequence, in each time window, thirty-six option values are used to estimate the twelve model parameters (i.e., $n_{P}=18$ put option prices and $n_{C}=18$ call option prices). In this way we generate a historical series of daily observations for each parameter. The date associated with the value of the parameters obtained in the $j$-th window is the date corresponding to the observed call and put option prices of the $j$-th window.

Figures 13 and 14 show the in-sample and out-of-sample option prices as a function of time to 

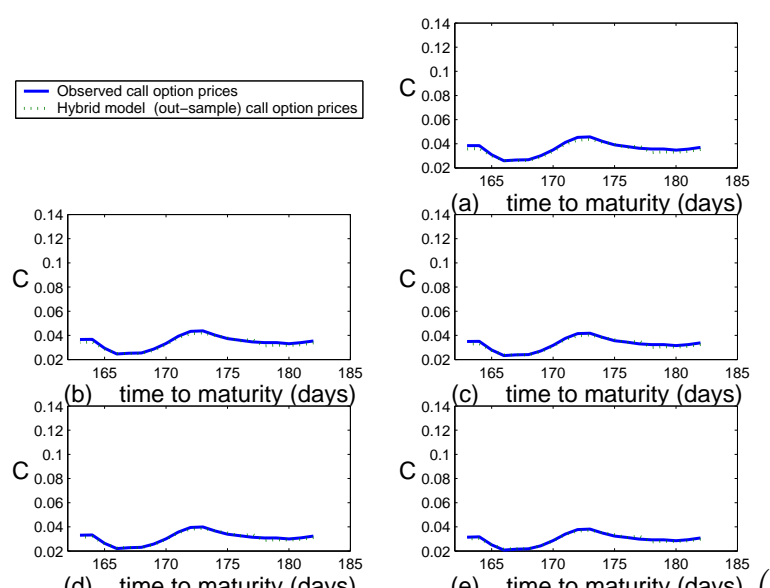

(d) time to maturity (days)

(e) time to maturity (days) (a)

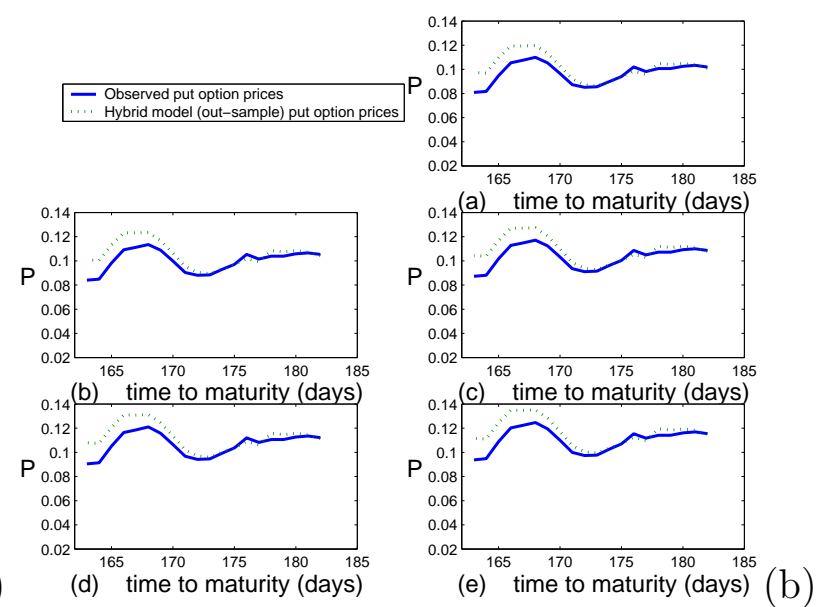

Figure 14: Out-of-sample observed and theoretical call (a) and put (b) option prices on YTU1 with strike price $E_{i}=1.375+0.005(i-1), i=1,2, \ldots, 18$, and expiry date $T=$ September 9,2011 versus time to maturity (December 20, 2010-January 14, 2011).
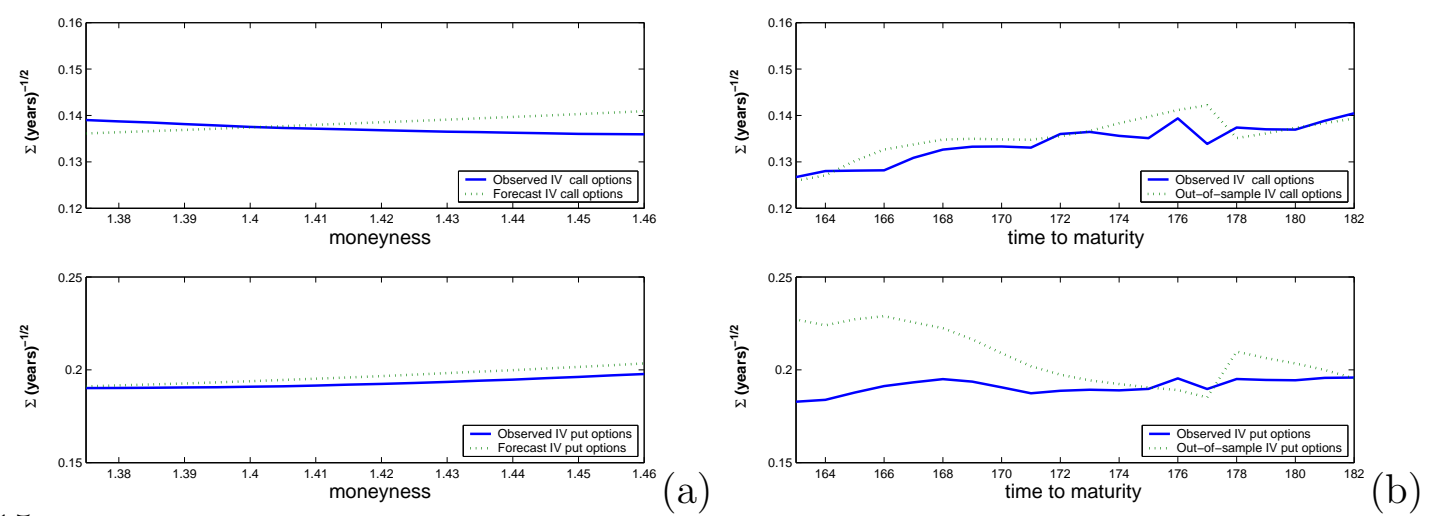

Figure 15: Left panel: time averaged out-of-sample implied volatility associated with observed and theoretical call (left upper panel) and put (left lower panel) option prices on YTU1 versus average moneyness $S_{t} / E, E=$ $E_{i}=1.375+0.005(i-1), i=1,2, \ldots, 18$ (expiry date $T=$ September 9, 2011). Right panel: strike average out-of-sample implied volatility associated with observed and theoretical call (right upper panel) and put (right lower panel) versus time to maturity (December 20, 2010 (184 days) - January 14, 2011 (163 days)).

maturity. We observe that the quality of the out-of-sample call option prices slightly outperforms that of the put option prices while the in-sample put option prices are more accurate than the in-sample call option prices. In fact, the sample means of the relative errors of the in-sample call and put options for the HW-Heston model are $3.21 \%$ and $1.49 \%$ respectively, while the sample mean of the relative errors of the out-of-sample call and put options are $5.01 \%$ and $5.76 \%$.

The left panels of Figure 15 show the time average out-of-sample implied volatility of call (upper panel) and put (lower panel) options as a function of the strike price. The right panels show the strike average out-of-sample implied volatility of call (upper panel) and put (lower panel) options as a function of time to maturity. Specifically, the $x$-axis value 183 (i.e., 183 days to maturity) corresponds to December 20, 2010 and the value 163 corresponds to January 14, 2011. We observe that the forecast values of the implied volatility are satisfactory up to ten days 

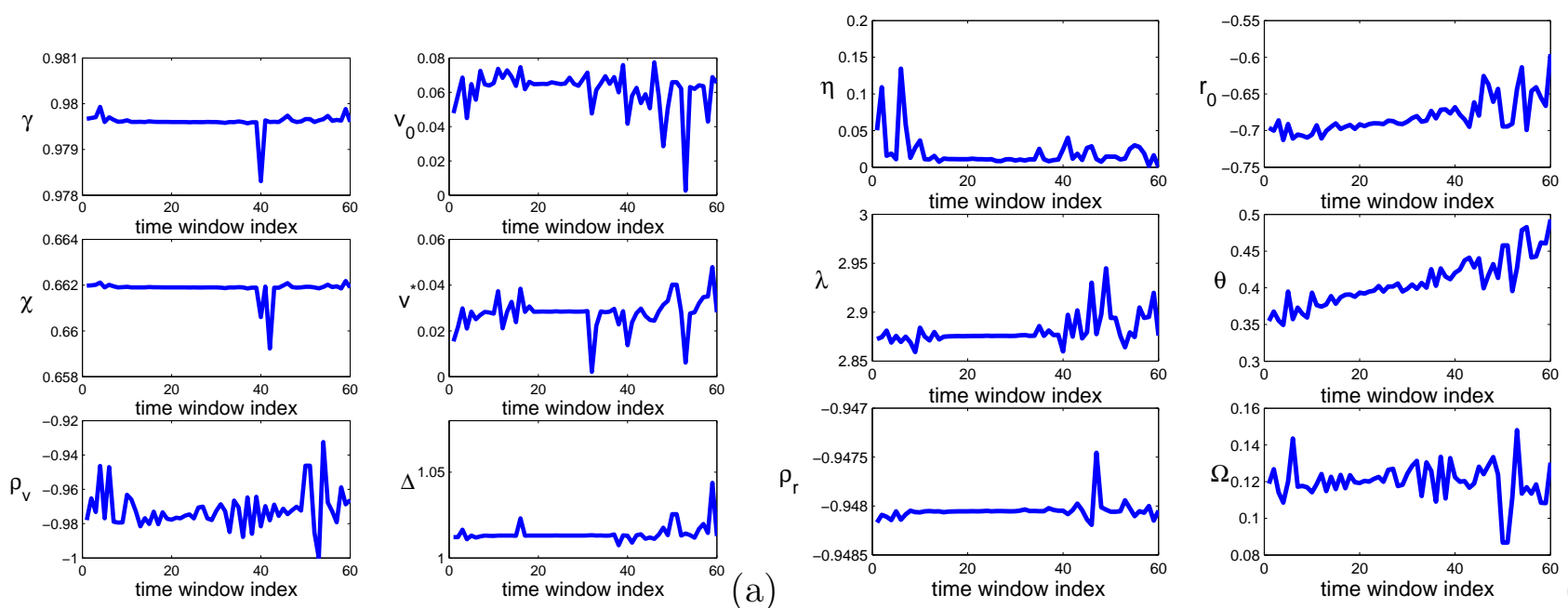

Figure 16: Estimated parameters $\gamma, v_{0}, \chi, v^{*}, \rho_{v}$, and $\Delta$ (panel (a)) and estimated parameters $\eta, r_{0}, \lambda, \theta, \rho_{r}$, and $\Omega$ (panel (b)) versus window index resulting from calibration of the HHW model to FX data.

from the last calibration, which was carried out on December 17, 2010.

Figure 16 shows the model parameters estimated using the FX data. We observe that $r_{0}$ is negative, while the long-term mean $\theta$ is a positive increasing function of time. The time series of the parameters related to the stochastic interest rate shows that in the last period corresponding to November-December 2010, there is turbulence in the time series of $r_{0}, \theta$. Finally, comparing the estimated values of $r_{0}$ obtained in the empirical analysis in Section 4.1 with those in this section (i.e., Figs. 5 and 16), we observe negative values of $r_{0}$. However, the values of $r_{0}$ corresponding to the period April--July 2012 are significantly less negative than those corresponding to the period January-July 2011. This agrees with the observed behavior of the short-term government bond yields (see Fig. 2), but the persistence of the negative sign may anticipate fluctuation and a new decrease in the yields.

\subsection{An empirical analysis of the U.S. three-month government bond yield against the S\&P 500 index}

In this section we use the HHW model to explore the dependence, if any, of the S\&P 500 index on the U.S. three-month government bond yield. In this analysis, the measure associated with the HHW model is the physical measure and the stochastic interest rate in Eq. (1) must be interpreted as the drift of the S\&P 500 index, which is assumed to be related to the U.S. threemonth government bond yield.

In this experiment, we calibrate the model parameters by solving

$$
\max _{\Theta \in \mathcal{V}} \mathcal{F}_{n_{T}}(\Theta)
$$


where $\mathcal{V}$ is given in (35), $n_{T}$ is the number of daily observations used, and $\mathcal{F}_{n_{T}}$ is the objective function (see Varin et al. 2011)

$$
\mathcal{F}_{n_{t}}=\sum_{j=1}^{n_{T}} D_{v, q}\left(x_{t_{1}}, v_{0}, r_{t_{1}}, t_{1}, x_{j+1}, r_{j+1}, t_{j+1}\right)
$$

Here, the function $D_{v, q}$ is given by (76), where $q$ is equal to zero, and $x_{j}, r_{j}$ are the observations of the S\&P 500 index and the U.S. three-month government bond yield respectively at $t=t_{j}$, $j=1,2, \ldots, n_{T}$. Note that in this experiment, $r_{0}$ is not estimated but is equal to $r_{t_{1}}$, that is, the observed value of the three-month government bond on the first day of the time window (i.e., $t=t_{1}$ ). We associate the last day of the time window, $t=t_{n_{T}}$, with each parameter value obtained by solving problem (39). In this way, we have a time series for each model parameter except for the initial stochastic interest rate, $r_{0}$, which is chosen to be the value of the three-month bond yield on the first day of the time window used in the calibration.

More specifically, we analyze the time series of daily observations of the U.S. S\&P 500 index and the U.S. three-month government bond yield in the period July 1, 2011-July 31, 2012 (see Figure 2). We estimate the model parameters (i.e., we solve 12 problems) by maximizing function (40) using $n_{T}=22$ daily data sets for the U.S. S\&P 500 index and the U.S. bond yield. After estimating the model parameters, we move this window along the time series, discarding the $n_{T}$ observations already used and inserting new $n_{T}$ observations. This procedure allows us to obtain a time series of monthly observations for each parameter, and the date associated with each estimated parameter is the last day of the time window, $t=t_{n_{T}}$, used in the estimation procedure. The historical series of the model parameters are indexed by integer values; the first one (i.e., $i=1$ ) corresponds to July 31, 2011 and the last one (i.e., $i=12$ ) corresponds to July $31,2012$.
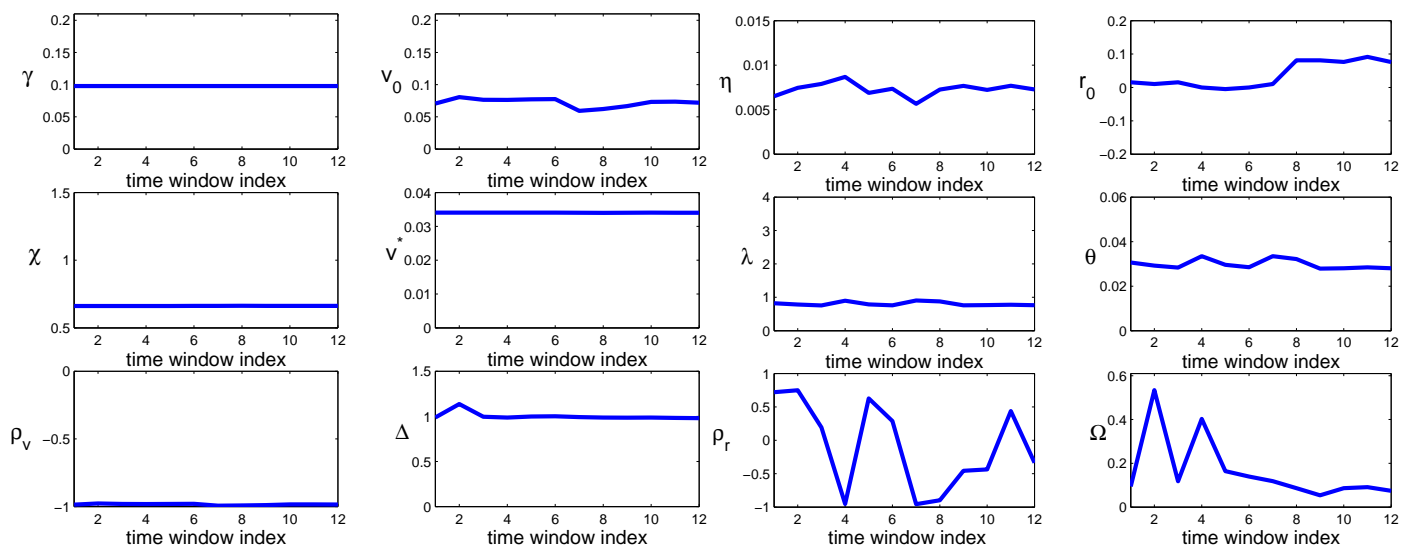

Figure 17: Estimated parameters of the volatility (left panels) and interest rate processes (right panels) using a time window of one month. The data used for calibration are daily observations of the U.S. three-month government bond yield (upper right panel) and the U.S. S\&P 500 index. 
Figure 17 shows the model parameters versus the window index. The values of the parameters related to the variance process behave like those in the experiment illustrated in Section 4.1 (see Figure 4), while the behavior of the estimated parameters, $\eta, \lambda, \rho_{r}, \Omega$, of the interest rate process differ from those shown in Figure 5. Specifically, the correlation coefficient, $\rho_{v}$, and the interest rate price volatility, $\Omega$, display strong fluctuations when the value of short-term bond yield is approximately zero. Comparing the right panel of Figure 2 and the behavior of $\Omega$ in the lower right panel of Figure 17, we see that the oscillations disappear when the window index is larger than $i=4$. In fact, $\Omega$ rapidly decreases in November and December 2011 and is practically constant in the period January 2012 to July 2012. The results of this experiment suggest that

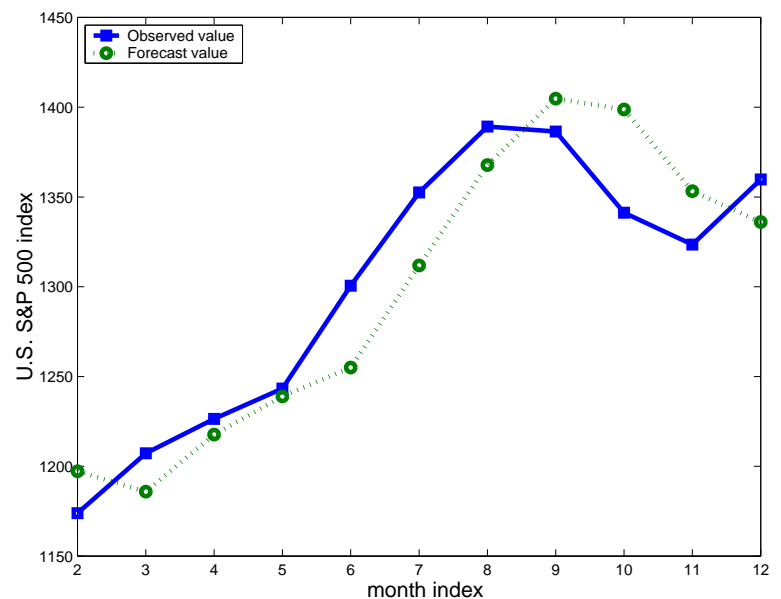

Figure 18: One-month ahead forecast of the S\&P 500 index. True values of the index (solid line) and model forecasts (dotted line) versus time index, $i=1$ : August, 30, 2011, $i=2$ : September 30, 2011, ., $i=11$ : August 30, 2012. The true values are monthly data freely downloadable from http://data.okfn.org/data/core/s-and-p$500 \#$ data.

when the short-term government bond yields are around zero, the behavior of the market index is strongly affected by the bond yield, while this effect decreases when the yields are around $0.1 \%$. These are only preliminary results which deserve further investigation. However, in order to assess the performance of this estimation, we use the estimated values of the parameters obtained at time $t$ to forecast the U.S. S\&P 500 index at time $t+\Delta t$, where $\Delta t=1$ month.

The forecast values of the S\&P 500 index are obtained using the expected value in formula (21) adapted to the current situation, that is:

$$
E\left(S_{t+\Delta t}\right)=S_{t} e^{r_{t} \Psi_{1, \lambda}(\Delta t)} e^{\left(\Delta t-\Psi_{1, \lambda}(\Delta t)\right)\left(\theta+\frac{\eta^{2}}{2 \lambda^{2}}+\frac{\eta}{\lambda} \Omega \rho_{r}\right)} e^{\frac{\eta^{2}}{2 \lambda^{2}}\left(\Psi_{2, \lambda}(\Delta t)-\Psi_{1, \lambda}(\Delta t)\right)} .
$$

The parameter values used here are those estimated in the time window $[t-\Delta t, t]$. Figure 18 displays the true values of the S\&P 500 index (solid line and squares) and the one-month ahead forecasts (dotted line and stars). The true data are monthly observations of the S\&P 500 index downloadable from the website http://data.okfn.org/data/core/s-and-p-500\#data. The average 
relative error is 0.024 and the median is 0.017 . As mentioned above, the results obtained are very preliminary, but they are encouraging.

\section{Conclusions}

This paper proposes a multi-factor Heston model (HHW model) that allows for stochastic volatility and a negative interest rate while preserving its analytical tractability. We derive integral representation formulas for the transition probability density function and for option pricing that involve one-dimensional integrals and elementary integrand functions as well as explicit formulas for the moments of the price variable. One of the main features of the model is that it allows for negative interest rates, and the first-order moment of the price variable depends on the correlation parameter between interest rate and asset price. The three empirical analyses illustrated in Section 4 assess the performance of the HHW in interpreting and forecasting option prices (see Sections 4.1 and 4.2) and asset prices (see Section 4.3) in a period where negative short-term government bond yields were experienced.

The model seems to capture the implied volatility of call and put options with only one set of estimated model parameters when we price both index and FX options. Furthermore, preliminary results from analysis of the relation between the S\&P 500 index and the short-term government bond show that the correlation parameter, $\rho_{r}$, and the interest rate price volatility, $\Omega$, strongly fluctuate when negative yields occur, while they are roughly constant when the yield moves away from zero. The construction of an early warning indicator of instability in short-term government bond yields using these parameters will be the object of further research.

\section{Acknowledgements}

The research leading to these results has received funding from the European Union, Seventh Framework Programme FP7, under grant agreement FinMaP no. 612955 and from the National Social Science Foundation of China, on the project of Evaluation and Research on the Policy Effectiveness of National "Famous Medical Studio" Based on the Inter-generational Transfer of Knowledge, under grant agreement no.15BGL158.

\section{Appendix: The analytical treatment of the HHW model}

In this section, we derive an integral representation formula for the transition probability density function, $p_{f}$, of the process in Eqs. (7)-(9) via a straightforward modification of the results illus- 
trated in Recchioni and Sun 2016. To this end, we recall that the function $p_{f}\left(x, v, r, t, x^{\prime}, v^{\prime}, r^{\prime}, t^{\prime}\right)$, $t<t^{\prime}$, as a function of the variables $(x, v, r, t)$ (i.e., the past variables, since $\left.t<t^{\prime}\right)$ satisfies the following backward Kolmogorov equation:

$$
\begin{aligned}
& -\frac{\partial p_{f}}{\partial t}=\frac{1}{2}\left(\tilde{\psi} v+\Omega^{2}\right) \frac{\partial^{2} p_{f}}{\partial x^{2}}+\frac{1}{2} \gamma^{2} v \frac{\partial^{2} p_{f}}{\partial v^{2}}+\frac{1}{2} \eta^{2} \frac{\partial^{2} p_{f}}{\partial r^{2}}+\gamma\left(\rho_{v}+\Delta\right) v \frac{\partial^{2} p_{f}}{\partial x \partial v} \\
& +\eta \rho_{r} \Omega \frac{\partial^{2} p_{f}}{\partial x \partial r}+\chi\left(v^{*}-v\right) \frac{\partial p_{f}}{\partial v}+\lambda(\theta-r) \frac{\partial p_{f}}{\partial r}+\left(r-\frac{1}{2}\left(\tilde{\psi} v+\Omega^{2}\right)\right) \frac{\partial p_{f}}{\partial x} \\
& (x, v, r) \in \mathbf{R} \times \mathbf{R}^{+} \times \mathbf{R}, 0 \leq t<t^{\prime},
\end{aligned}
$$

where $\tilde{\psi}$ is given in (10), with final condition

$$
\begin{gathered}
p_{f}\left(x, v, r, t^{\prime}, x^{\prime}, v^{\prime}, r^{\prime}, t^{\prime}\right)=\delta\left(x^{\prime}-x\right) \delta\left(v^{\prime}-v\right) \delta\left(r^{\prime}-r\right), \\
(x, v, r),\left(x^{\prime}, v^{\prime}, r^{\prime}\right) \in \mathbf{R} \times \mathbf{R}^{+} \times \mathbf{R}, t^{\prime} \geq 0,
\end{gathered}
$$

and appropriate boundary conditions. Since neither the coefficients (42) nor the final condition (43) depend on translation in the log-price variable, we can proceed as in Recchioni and Sun 2016, assuming the following representation formula for $p_{f}$ :

$$
\begin{aligned}
& p_{f}\left(x, v, r, t, x^{\prime}, v^{\prime}, r^{\prime}, t^{\prime}\right)= \\
& \frac{e^{q\left(x-x^{\prime}\right)}}{2 \pi} \int_{\mathbf{R}} e^{\imath k\left(x^{\prime}-x\right)} \frac{1}{(2 \pi)^{2}} \int_{\mathbf{R}} e^{\imath l v^{\prime}} \int_{\mathbf{R}} e^{\imath \xi r^{\prime}} e^{A(\tau, k, l, \xi)} e^{-v B_{v}(\tau, k, l)} e^{-r B_{r}(\tau, k, \xi)} d l d \xi d k,
\end{aligned}
$$

where $\tau=t^{\prime}-t$. Substituting formula (44) into equation (42), functions $A, B_{v}$, and $B_{r}$ must satisfy the following ordinary differential equations:

$$
\begin{gathered}
\frac{d A}{d \tau}(\tau, k, l, \xi)= \\
-\lambda \theta B_{r}(\tau, k, \xi)-\chi v^{*} B_{v}(\tau, k, l)+\left(-\varphi_{q}(k) \Omega^{2}+(\imath k-q) \Omega \rho_{p, r} \eta B_{r}(\tau, k, \xi)+\frac{\eta^{2}}{2} B_{r}^{2}(\tau, k, \xi)\right) \\
=-\varphi_{q}(k) \Omega^{2}-\left[\lambda \theta-(\imath k-q) \Omega \rho_{p, r} \eta\right] B_{r}(\tau, k, \xi)+\frac{\eta^{2}}{2} B_{r}^{2}(\tau, k, \xi)-\chi v^{*} B_{v}(\tau, k, l) \\
\frac{d B_{v}}{d \tau}(\tau, k, l)=\varphi_{q}(k) \tilde{\psi}-\left(\chi+(\imath k-q) \gamma\left(\rho_{v}+\Delta\right)\right) B_{v}(\tau, k, l)-\frac{\gamma^{2}}{2} B_{v}^{2}(\tau, k, l) \\
\frac{d B_{r}}{d \tau}(\tau, k, \xi)=(\imath k-q)-\lambda B_{r}(\tau, k, \xi)
\end{gathered}
$$

with initial conditions

$$
A(0, k, l, \xi)=0, \quad B_{v}(0, k, l)=\imath l, \quad B_{r}(0, k, \xi)=\imath \xi
$$


where $\varphi_{q}$ is the following quantity:

$$
\varphi_{q}(k)=\frac{k^{2}}{2}+\imath \frac{k}{2}(2 q-1)-\frac{1}{2}\left(q^{2}-q\right), k \in \mathbf{R} .
$$

The solution to problem (46), (48), which is given in Recchioni and Sun 2016, is

$$
B_{v}(\tau, k, l)=\frac{2}{\gamma^{2}} \frac{\frac{d}{d \tau} C_{v}}{C_{v}}
$$

where

$$
C_{v}(\tau, k, l)=e^{\left(\mu_{q, v}+\zeta_{q, v}\right) \tau}\left[\frac{s_{q, v, b}+\imath l \frac{\gamma^{2}}{2} s_{q, v, g}}{2 \zeta_{q, v}}\right],
$$

so we have

$$
B_{v}(\tau, k, l)=\frac{2}{\gamma^{2}} \frac{\left(\left(\zeta_{q, v}^{2}-\mu_{q, v}^{2}\right) s_{q, v, g}+\frac{\gamma^{2}}{2} l l s_{q, v, d}\right)}{s_{q, v, b}+\imath l \frac{\gamma^{2}}{2} s_{q, v, g}}
$$

where

$$
\begin{aligned}
\mu_{q, v} & =-\frac{1}{2}\left(\chi+(\imath k-q) \gamma\left(\rho_{v}+\Delta\right)\right), \\
\zeta_{q, v} & =\frac{1}{2}\left[4 \mu_{v}^{2}+2 \gamma^{2} \varphi_{q}(k) \tilde{\psi}\right]^{1 / 2}, \\
s_{q, v, g} & =1-e^{-2 \zeta_{q, v} \tau}, \\
s_{q, v, b} & =\left(\zeta_{q, v}+\mu_{q, v}\right) e^{-2 \zeta_{q, v} \tau}+\left(\zeta_{q, v}-\mu_{q, v}\right),
\end{aligned}
$$

and $s_{q, v, d}$ is given by

$$
s_{q, v, d}=\left(\zeta_{q, v}-\mu_{q, v}\right) e^{-2 \zeta_{q, v} \tau}+\left(\zeta_{q, v}+\mu_{q, v}\right) .
$$

The solution of Eq. (47) is given by:

$$
B_{r}(\tau, k, \xi)=\imath \xi e^{-\lambda \tau}+(\imath k-q) \psi_{1, \lambda}(\tau),
$$

where $\Psi_{1, \lambda}$ is given by Eq. (19). Finally, integrating Eq. (45), we obtain:

$$
A(\tau, k, l, \xi)=-\xi^{2} Q_{2}(\tau)+\imath \xi Q_{1}(\tau, k)+Q_{0}(\tau, k)-2 \frac{\chi v^{*}}{\gamma^{2}} \ln C_{v},
$$

where $Q_{0}, Q_{1}$ and $Q_{2}$ are given by

$$
\begin{aligned}
& Q_{0}(\tau, k)=-\varphi_{q}(k) \Omega^{2} \tau-a_{q, r}(k) \frac{(\imath k-q)}{\lambda}\left(\tau-\Psi_{1, \lambda}(\tau)\right)+\frac{\eta^{2}}{2} \frac{(\imath k-q)^{2}}{\lambda^{2}}\left(\tau-2 \Psi_{1, \lambda}(\tau)+\Psi_{2, \lambda}(\tau)\right), \\
& Q_{1}(\tau, k)=-a_{q, r} \Psi_{1, \lambda}(\tau)+\eta^{2}\left(\frac{\imath k-q}{\lambda}\right)\left(\Psi_{1, \lambda}(\tau)-\Psi_{2, \lambda}(\tau)\right), \\
& Q_{2}(\tau)=\frac{\eta^{2}}{2} \Psi_{2, \lambda}(\tau),
\end{aligned}
$$


while functions $\Psi_{j, \lambda}, j=1,2$ are given in (19) and $a_{q, r}$ is given by

$$
a_{q, r}=\lambda \theta-(\imath k-q) \Omega \rho_{r} \eta
$$

By using the specific form of functions $A, B_{r}$, and $B_{v}$, we can rewrite the integrand appearing in Eq. (44) as the product of two functions, one of which depends only on the parameters of the volatility process and the other on the interest rate process:

$$
e^{A(\tau, k, l, \xi)} e^{-v B_{v}(\tau, k, l)} e^{-r B_{r}(\tau, k, \xi)}=e^{\Psi_{v}(\tau, k, l, v)} e^{\Psi_{r}(\tau, k, \xi, r)}
$$

where

$$
\begin{aligned}
& \Psi_{v}(\tau, k, l, v)=-\frac{2 \chi v^{*}}{\gamma^{2}} \ln C_{v}(\tau, k, l)-\frac{2 v}{\gamma^{2}} \frac{1}{C_{v}} \frac{d C_{v}}{d \tau}(\tau, k, l) \\
& \Psi_{r}(\tau, k, \xi, r)=-\xi^{2} Q_{2}(\tau)+\imath \xi\left(Q_{1}(\tau, k)-r e^{-\lambda \tau}\right)+Q_{0}(\tau, k)-r(\imath k-q) \Psi_{1, \lambda} .
\end{aligned}
$$

In order to obtain an explicit expression for $p_{f}$, we compute the following two inverse Fourier transforms:

$$
\begin{aligned}
& L_{v, q}\left(\tau, v, v^{\prime}, k ; \Theta_{v}\right)=\frac{1}{2 \pi} \int_{-\infty}^{+\infty} e^{\imath l v^{\prime}} e^{\Psi_{v}(\tau, k, l, v)} d l \\
& L_{r, q}\left(\tau, r, r^{\prime}, k ; \Theta_{r}\right)=\frac{1}{2 \pi} \int_{-\infty}^{+\infty} e^{\imath \xi r^{\prime}} e^{\Psi_{r}(\tau, k, \xi, r)} d \xi
\end{aligned}
$$

The first function, $L_{v, q}$, is given by (see Recchioni and Sun 2016 for further details)

$$
\begin{aligned}
& L_{v, q}\left(\tau, v, v^{\prime}, k ; \Theta_{v}\right)=e^{-\left(2 \chi v^{*} / \gamma^{2}\right)\left[\ln \left(s_{q, v, b} / 2 \zeta_{q, v}\right)+\left(\mu_{q, v}+\zeta_{q, v}\right) \tau\right]} e^{-\left(2 v / \gamma^{2}\right)\left(\zeta_{q, v}^{2}-\mu_{q, v}^{2}\right) s_{q, v, g} / s_{q, v, b}} \times \\
& M_{q, v}\left(M_{q, v} \tilde{v}_{q}\right)^{-\nu_{v} / 2}\left(M_{q, v} v^{\prime}\right)^{\nu_{v} / 2} e^{-M_{q, v} \tilde{v}_{q}} e^{-M_{q, v} v^{\prime}} I_{\nu_{v}}\left(2 M_{q, v}\left(\tilde{v}_{q} v^{\prime}\right)^{1 / 2}\right)
\end{aligned}
$$

with $\nu_{v}=\left(2 \chi v^{*} / \gamma^{2}\right)-1$ and $M_{q, v}, \tilde{v}_{q}$ given by

$$
M_{q, v}=\frac{2}{\gamma^{2}} \frac{s_{q, v, b}}{s_{q, v, g}}, \quad \tilde{v}_{q}=\frac{4 \zeta_{q, v}^{2} v e^{-2 \zeta_{q, v} \tau}}{s_{q, v, b}^{2}}, \quad M_{q, v} \tilde{v}_{q}=\frac{8}{\gamma^{2}} \frac{\zeta_{q, v}^{2} v e^{-2 \zeta_{q, v} \tau}}{s_{q, v, g} s_{q, v, b}} .
$$

The quantities $\zeta_{q, v}, \mu_{q, v}, s_{q, v, b}, s_{q, v, g}$ appearing in (69) and (70) are given in (53)-(56).

The second function, $L_{r, q}$, can be easily obtained by integrating the Gaussian integral:

$$
L_{r, q}\left(\tau, r, r^{\prime}, k ; \Theta_{r}\right)=e^{-r(\imath k-q) \Psi_{1, \lambda}(\tau)} \frac{e^{Q_{0}(\tau, k)}}{2 \sqrt{\pi Q_{2}(\tau)}} e^{-\frac{1}{4 Q_{2}(\tau)}\left(r^{\prime}+Q_{1}(\tau, k)-r e^{-\lambda \tau}\right)^{2}} .
$$

Moreover, we have:

$$
\int_{0}^{+\infty} L_{v, q}\left(\tau, v, v^{\prime}, k ; \Theta_{v}\right) d v^{\prime}=e^{Q_{v, q}\left(\tau, v, k ; \Theta_{v}\right)}
$$


and

$$
\int_{-\infty}^{+\infty} e^{-\omega T r^{\prime}} L_{r, q}\left(\tau, r, r^{\prime}, k ; \Theta_{r}\right) d r^{\prime}=e^{Q_{r, q}\left(\tau, r, k ; \Theta_{r}\right)} e^{\omega T\left(Q_{1}(T, K)-r e^{-\lambda T}\right)} e^{(\omega T)^{2} Q_{2}(T)}
$$

where $Q_{v, q}, Q_{r, q}$ are given by

$$
\begin{gathered}
Q_{v, q}\left(\tau, v, k ; \Theta_{v}\right)=\frac{-2 \chi v^{*}}{\gamma^{2}} \ln \left(s_{q, v, b} /\left(2 \zeta_{q, v}\right)\right)-\frac{2 \chi v^{*}}{\gamma^{2}}\left(\zeta_{q, v}+\mu_{q, v}\right) \tau-\frac{2 v}{\gamma^{2}}\left(\zeta_{q, v}^{2}-\mu_{q, v}^{2}\right) \frac{s_{q, v, g}}{s_{q, v, b}}, \\
Q_{r, q}\left(\tau, r, k ; \Theta_{r}\right)=-r(\imath k-q) \Psi_{1, \lambda}(\tau)+Q_{0}(\tau, k),
\end{gathered}
$$

where $\omega$ is a positive constant and $Q_{0}, Q_{1}, Q_{2}$ are the quantities given by (60)-(62), while $\zeta_{q, v}$, $\mu_{q, v}, s_{q, v, b}, s_{q, v, g}$ are given by (53)-(56) and $\Psi_{j, \lambda}, j=1,2$ is given by (19).

From (72) and (73) and the explicit formula (12) for $p_{f}$, we obtain an integral representation formula for the marginal probability density function, $D_{v, q}\left(x, r, t, x^{\prime}, r^{\prime}, t^{\prime}\right)$, of the future variables $\left(x^{\prime}, r^{\prime}\right)$ :

$$
\begin{gathered}
D_{v, q}\left(x, r, t, x^{\prime}, r^{\prime}, t^{\prime}\right)=\int_{0}^{+\infty} p_{f}\left(x, v, r, t, x^{\prime}, v^{\prime}, r^{\prime}, t^{\prime}\right) d v^{\prime} \\
=\frac{e^{q\left(x-x^{\prime}\right)}}{2 \pi} \int_{-\infty}^{+\infty} e^{\imath k\left(x^{\prime}-x\right)} e^{Q_{v, q}\left(t^{\prime}-t, v, k ; \Theta_{v}\right)} L_{r, q}\left(t^{\prime}-t, r, r^{\prime}, k ; \Theta_{r}\right) d k
\end{gathered}
$$

as well as for the marginal probability density function, $D_{v, r, q}\left(x, t, x^{\prime}, t^{\prime}\right)$, of the price variable $x^{\prime}$ :

$$
\begin{aligned}
& D_{v, r, q}\left(x, t, x^{\prime}, t^{\prime}\right)=\int_{-\infty}^{+\infty} \int_{0}^{+\infty} p_{f}\left(x, v, r, t, x^{\prime}, v^{\prime}, r^{\prime}, t^{\prime}\right) d r^{\prime} d v^{\prime} \\
= & \frac{e^{q\left(x-x^{\prime}\right)}}{2 \pi} \int_{-\infty}^{+\infty} e^{\imath k\left(x^{\prime}-x\right)} e^{Q_{v, q}\left(t^{\prime}-t, v, k ; \Theta_{v}\right)} e^{Q_{r, q}\left(t^{\prime}-t, r, k ; \Theta_{r}\right)} d k
\end{aligned}
$$

where the functions $Q_{v, q}, Q_{r, q}$ are given in (74) and (75).

Let us show that the function $p_{f}$, obtained by solving problem (42), (43), is a transition probability density function. As shown in Craddock 2009, this is necessary since fundamental solutions of Kolmogorov equations are not necessarily transition probability density functions.

To this end, we first compute the integral of $p_{f}$ with respect to the future variable $x^{\prime}$. Using the following formula for the Dirac delta function (see Jacquier and Lorig 2013 for further applications of this formula):

$$
\frac{1}{2 \pi} \int_{-\infty}^{+\infty} e^{\imath\left(x^{\prime}-x\right)(k+\imath q)} d x^{\prime}=\delta(k+\imath q)
$$

we obtain

$$
\int_{-\infty}^{+\infty} p_{f}\left(x, v, r, t, x^{\prime}, v^{\prime}, r^{\prime}, t^{\prime}\right) d x^{\prime}=L_{v, q}\left(t^{\prime}-t, v, v^{\prime},-\imath q ; \Theta_{v}\right) L_{r, q}\left(t^{\prime}-t, r, r^{\prime},-\imath q ; \Theta_{r}\right)
$$


where $L_{v, q}\left(t^{\prime}-t, v, v^{\prime},-\imath q, \Theta_{v}\right)$ is the transition probability density function of the CIR model (see Craddock 2009 Eq. (3.4)). In fact, by setting $k=-\imath q$ in Eqs. (69) and (53)-(55), we obtain

$$
L_{v, q}\left(\tau, v, v^{\prime},-\imath q ; \Theta_{v}\right)=\frac{2}{\gamma^{2}} \frac{\chi}{1-e^{-\chi \tau}} \frac{v^{\nu_{v} / 2}}{\left(v e^{-\chi \tau}\right)^{\nu / 2}} e^{-2 \frac{\chi}{1-e^{-\chi \tau}}\left(v e^{-\chi \tau}+v^{\prime}\right)} .
$$

Similarly, by setting $k=-\imath q$ in Eqs. (61), (60), and (71), $L_{r, q}\left(t^{\prime}-t, r, r^{\prime} ;-\imath q, \Theta_{r}\right)$ is the following Gaussian transition probability density function:

$$
L_{r, q}\left(\tau, r, r^{\prime},-\imath q ; \Theta_{r}\right)=\frac{1}{2 \sqrt{\pi Q_{2}(\tau)}} e^{-\frac{1}{4 Q_{2}(\tau)}\left(r^{\prime}-r e^{-\lambda \tau}-\lambda \theta \Psi_{1, \lambda}(\tau)\right)^{2}}
$$

Using Eqs. (79)-(81), we have

$$
\int_{-\infty}^{\infty} \int_{0}^{\infty} \int_{-\infty}^{\infty} p_{f}\left(x, v, r, t, x^{\prime}, v^{\prime}, r^{\prime}, t^{\prime}\right) d x^{\prime} d r^{\prime} d v^{\prime}=1, \forall x, v, r, t, t^{\prime} .
$$

We conclude this Appendix by deducing explicit formulas for the moments of the price variable; specifically, we use formulas (76) and (77). In fact, the formula for the $m^{\text {th }}$ moment of the price $S_{t^{\prime}}=S_{0} e^{x^{\prime}}$ conditioned to the observation at time $t=0$ is given by the following integral:

$$
\begin{aligned}
& \mathcal{M}_{m}\left(S_{0}, v_{0}, r_{0}\right)=E\left(S_{t^{\prime}}^{m}\right)=S_{0}^{m} \int_{-\infty}^{+\infty} e^{m x^{\prime}} D_{v, r, q}\left(0, v_{0}, r_{0}, 0, x^{\prime}, t^{\prime}\right) d x^{\prime} \\
& =S_{0}^{m} \frac{1}{2 \pi} \int_{-\infty}^{+\infty}\left(\int_{-\infty}^{+\infty} e^{m x^{\prime}} e^{-q x^{\prime}} e^{\imath k x^{\prime}}\right) e^{Q_{v, q}\left(t^{\prime}, v_{0}, k ; \Theta_{v}\right)} e^{Q_{r, q}\left(t^{\prime}, r_{0}, k ; \Theta_{r}\right)} d k d x^{\prime} .
\end{aligned}
$$

Setting $q=m$, the integral in the parentheses in Eq. (83) yields the Dirac delta function concentrated at $k=0$, which allows us to deduce the following formula for the moments:

$$
\mathcal{M}_{m}=E\left(S_{t^{\prime}}^{m}\right)=S_{0}^{m} e^{Q_{v, m}\left(t^{\prime}, v_{0}, 0 ; \Theta_{v}\right)} e^{Q_{r, m}\left(t^{\prime}, r_{0}, 0 ; \Theta_{r}\right)}
$$

where $Q_{v, m}, Q_{r, m}$ are the functions given in Eqs. (74) and (75) with $q=m$ and $k=0$. We note that formula (84) is an elementary formula that does not involve integrals and it may be useful for investigating the correlation between interest rates and market indices/assets. Finally, by taking the limit $\Omega \rightarrow 0^{+}, \lambda \rightarrow 0^{+}$, and $\eta \rightarrow 0^{+}$in formula (84), we obtain the formula for the $m^{\text {th }}$ moment, $\mathcal{M}_{m}^{H}$ in the Heston framework.

\section{References}

[1] Ayache, E., Henrotte, P., Nassar, S., Wang, X. (2007). Can anyone solve the smile problem? Wilmott Magazine, Mar 2007, 78-96.

[2] Ahlip, R. (2008). Foreign exchange options under stochastic volatility and stochastic interest rates. International Journal of Theoretical and Applied Finance, 11, 277-294. 
[3] Andreasen, J. (2007). Closed form pricing of FX options under stochastic rates and volatility. International Journal of Theoretical and Applied Finance, 11(3), 277-294.

[4] Benhamou, E., Gobet E., and Miri M. (2012) Analytical formulas for local volatility model with stochastic rates. Quantitative Finance, 12(2), 185-198.

[5] Black, F., Scholes, M. (1973). The pricing of options and corporate liabilities. Journal of Political Economy, 81, 637-659.

[6] Bühler, H. (2002). Applying stochastic volatility models for pricing and hedging derivatives. Global Quantitative Research Deutsche Bank AG Global Equities. Freely downloadable from the website:

http://www.quantitative-research.de/dl/021118SV.pdf

[7] Christoffersen, P., Heston, S., Jacob, K. (2009). The shape and term structure of the index option smirk: Why multifactor stochastic volatility models work so well. Management Science 55, 1914-1932.

[8] Cieslak, A., Povala, P. (2014) Information in the Term Structure of Yield Curve Volatility. Journal of Finance, Forthcoming. Available at SSRN: 1458006

[9] Craddock, M. (2009) Fundamental solutions, transition densities and the integration of Lie symmetries, Journal of Differential Equations, 246, 2538-2560.

[10] Choi, Y., Wirjanto, T.S. (2007). Ananalytic approximation formula for pricing zero coupon bonds. Finance Research Letters, 4, 116-126.

[11] Deelstra, G., Rayee, G. (2012). Local volatility pricing models for long-dated FX derivatives. Applied Mathematical Finance, 20(4), 380-402.

[12] Duffie, D. (2001). Asset pricing theory. Princeton, New Jersey, USA: Princeton University Press.

[13] Duffie, D., Pan, J., Singleton, K. (2000). Transform analysis and asset pricing for affine jump diffusions. Econometrica, 68, 1343-1376.

[14] Dupire, B. (1994). Pricing with a smile. Risk Magazine, Jan 1994, 18-20.

[15] Fatone, L., Mariani, F., Recchioni, M.C., Zirilli, F. (2009). An explicitly solvable multi-scale stochastic volatility model: option pricing and calibration. Journal of Futures Markets, 29(9), 862-893. 
[16] Fatone, L., Mariani, F., Recchioni, M.C., Zirilli, F. (2013). The analysis of real data using a multiscale stochastic volatility model. European Financial Management, 19(1), 153-179 .

[17] Grzelak, L.A., Oosterlee C. W., Van Weeren, S. (2012). Extension of stochastic volatility equity models with Hull-White interest rate process. Quantitative Finance, 12, 89-105.

[18] Grzelak, L.A., Oosterlee C. W. (2011). On the Heston model with stochastic interest rates. SIAM Journal on Financial Mathematics, 2, 255-286.

[19] Hagan, P., Kumar, D., Lesniewski, A.S., Woodward, D.E. (2002). Managing smile risk. Wilmott Magazine, July 2002, 86-108.

[20] Heston, S. L. (1993). A closed-form solution for options with stochastic volatility with applications to bond and currency options. The Review of Financial Studies, 6, 327-343.

[21] Homescu, C. (2014). Local stochastic volatility models: calibration and pricing. SSRN:2448098.

[22] Hull, J., White, H. (1988). The pricing of options on assets with stochastic volatilities. Journal of Finance, 3, 327-343.

[23] Islyaev, S., Date, P. (2015). Electricity futures price models: Calibration and forecasting. European Journal of Operational Research 247, 144-154.

[24] Itkin, A. (2016). LSV models with stochastic interest rates and correlated jumps. International Journal of Computer Mathematics, DOI: 10.1080/00207160.2016.1188923.

[25] Jackson, H. (2015). The International Experience with Negative Policy Rates. Bank of Canada Staff Discussion Paper 2015-13.

[26] Jacquier, A., Lorig, M. (2013) The Smile of Certain Lévy-Type Models, SIAM Journal on Finanancial Mathematics 4(1), 804-830.

[27] Lipton, A. (2001). Mathematical methods for foreign exchange. Singapore: World Scientific Pubblishing Co. Pte. Ltd.

[28] Mamon, R.S. (2004). Three Ways to Solve for Bond Prices in the Vasicek Model. Journal of Applied Mathematics and Decision Sciences 8(1), 1-14.

[29] Pacati, C., Pompa, G., Renò, R. (2016). Smiling Twice: The Heston++ Model. Available at SSRN: 2697179 
[30] Pun, C.S., Chung, S.F., Wong, H.Y. (2015). Variance swap with mean reversion, multifactor stochastic volatility and jumps. European Journal of Operational Research 245, 571-580.

[31] Recchioni, M.C., Scoccia, A. (2000). A stochastic algorithm for constrained global optimization. Journal of Global Optimization, 16, 257-270.

[32] Recchioni, M.C., Sun, Y. (2016). An explicitly solvable Heston model with stochastic interest rate, European Journal of Operational Research 249, 2016, 359-377.

[33] Schoutens, W. (2003). Lévy processes in finance. Chichester, UK: Wiley \& Sons.

[34] Stein, E. M., J. C. Stein, J. C. (1991). Stock price distributions with stochastic volatility: an analytic approach. The Review of Financial Studies, 4, 727-752.

[35] Trolle, A. B., Schwartz, E.S. (2009). A general stochastic volatility model for the pricing of interest rate derivatives. The Review of Financial Studies, 22(5), 2007-2057.

[36] Varin, C., Reid, N., Firth, D. (2011). An overview of composite likelihood methods. Statistica Sinica, 21, 5-42

[37] Vasicek, O. (1977). An equilibrium characterization of the term structure, Journal of Financial Economics, 5, 177-188.

[38] Wong, H.Y., Lo, Y.W. (2009). Option pricing with mean reversion and stochastic volatility. European Journal of Operational Research 197, 179-187.

[39] Zhu, J. (2000). Modular Pricing of Options. Springer Verlag, Berlin. 